\title{
La bédé exposée au musée : un défi à relever
}

Chris Reyns-Chikuma, Université de l'Alberta

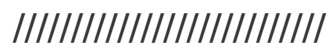

Tout a commencé avec un défi. Connaissant ma passion pour la bande dessinée (BD, bédé, comics, graphic novels/romans graphiques, manga), certains membres de la bibliothèque Rutherford de l'Université de l'Alberta (UofA) m'ont proposé d'organiser une exposition sur la BD. Je trouvais cela intéressant et j'ai accepté, mais comment allais-je faire ? Exposer quoi ? Des livres ? Dans des vitrines ? En quoi est-ce différent de ce que la bibliothèque fait déjà : mettre des livres à la disposition du public. Ceci se fait en effet depuis longtemps dans les bibliothèques publiques, d'abord à travers un catalogue (aujourd'hui en ligne et donc à un clic de distance), ensuite on va soi-même dans les rayons pour le/s consulter. Mieux encore aujourd'hui, de plus en plus, on peut accéder au livre directement sur son ordinateur (e-book/livre-é[lectronique]).

Donc il fallait autre chose de plus original et de plus engageant.

J'avais évidemment vu des expositions de BD et des catalogues d'exposition et je savais que l'on pouvait aussi exposer des copies de pages de BD sur les murs de diverses manières (encadrées, agrandies, etc.). Oui, mais comment choisir? Quelles pages ? Et comment les montrer ? En quel format? Plus grand que nature sans doute, car la distance entre l'image et le spectateurlecteur serait nécessairement plus grande que celle qui sépare le livre et le lecteur, mais comment expliquer cette page artistiquement, dans sa spécificité BD (non seulement le dessin, mais aussi les cases_-ses formes, ses bulles ou leur absence) ? Comment situer cette page dans sa continuité narrative puisque la BD est d'abord un art narratif ?

Des originaux ? C'est tentant et attirant. Mais c'est très difficile à obtenir, surtout aujourd'hui alors que les prix s'envolent (un dessin de Hergé est parti à 1,55 million d'euros à Paris en novembre 2016) et que j'avais un budget minuscule.

Je suis donc parti avec l'idée d'ajouter une dimension plus novatrice et plus dynamique où les visiteurs seraient plus sollicités, des « spectaCteurs » et «spectaCtrices ».

Avec les conseils d'un de mes collègues, Sean Caulfield, professeur d'art et artiste (et donc qui avait une grande expérience professionnelle des expositions), j'ai donc écrit et lancé l'appel à contribution créative que voici : 


\section{Appel à contributions ... créatives}

Wow ! Boum ! ドッカーン! La BD dans le musée : un défi !?

La bande dessinée a été exposée dans les musées depuis au moins 50 ans. La première exposition a eu lieu en France en 1966, et beaucoup d'autres ont suivi (New York, Angoulême, etc., et récemment à Glasgow). Jusqu'à tout récemment, les façons de montrer les BD dans les musées ont été assez traditionnelles, en suivant les règles muséologiques conventionnelles qui sont celles pour exposer les peintures et tout autre artefact « exposable» (sculptures, etc.). Cependant, contrairement à la peinture, la BD est un art spécifique qui n'est pas fait pour être accroché au mur. C'est pourquoi pendant cette nouvelle exposition nous proposons, nous essayons de répondre à cette question de manières diverses et concrètes : comment exposer des bandes dessinées dans un espace public?

Dans notre exposition, alors que nous aurons aussi quelques bandes-strips, pages et cases agrandies d'une manière conventionnelle sur les murs, nous montrerons les BD de manières qui mettent en évidence les spécificités de ce medium comme la séquentialité, le rôle et la variété des bulles-phylactères, des onomatopées, etc. Nous ajouterons aussi quelques reproductions de cases et pages de $\mathrm{BD}$ qui se passent entièrement ou partiellement dans un musée, ainsi que quelques photos de musées de la BD/Comics/Manga dans le monde, comme à Angoulême, Bruxelles, Kyoto, ou New York.

Mais tout aussi important, l'exhibition montrera VOTRE œuvre créative.

Nous voulons donc vous inviter, étudiants, chercheurs et artistes, à nous envoyer un travail artistique qui défie, « gentiment » ou « sauvagement » les techniques habituelles pour montrer des $\mathrm{BD}$, soit dans la tradition picturale comme les artistes pop tel que Lichtenstein, ou par un vidéo clip, ou une courte performance, ou toute autre forme et tout autre média. L'œuvre peut aussi refléter une pratique créative qui est motivée formellement ou conceptuellement par la tradition de la BD mais qui n'est pas nécessairement elle-même une BD au sens traditionnel du terme (comme certaines œuvres de l'artiste Marcel Dzama par exemple)

\section{Date limite}

$1^{\text {er }}$ juillet 2016

Une proposition de 100 mots, expliquant ce que sera votre projet (quel médium/média, quel type de défi, ...) 
A Envoyer à Chris Reyns-Chikuma (reynschi@ualberta.ca) et Sean Caulfield (stc@ualberta.ca)

\section{Exposition}

19 septembre -3 octobre 2016

Librairie Rutherford, Université de l'Alberta, Edmonton, Canada

Livraison de l'œuvre, prête à être exposée, pour le 15 août 2016 midi,

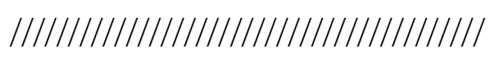

Les organisateurs se réservent le droit de rejeter toute œuvre qui n'est pas proprement préparée pour l'installation ou qui n'est pas liée au thème original de l'exposition.

$/ / / / / / / / /$

\section{Prix \$}

Top $3-100 \$$ chacune

Trois œuvres seront sélectionnées par un jury et par vote populaire pour un prix (100\$ chacune) et exposées à l'AGA (Galerie d'Art de l'Alberta) pendant un mois supplémentaire.

\section{////////////////////////////////////////////////////////////}

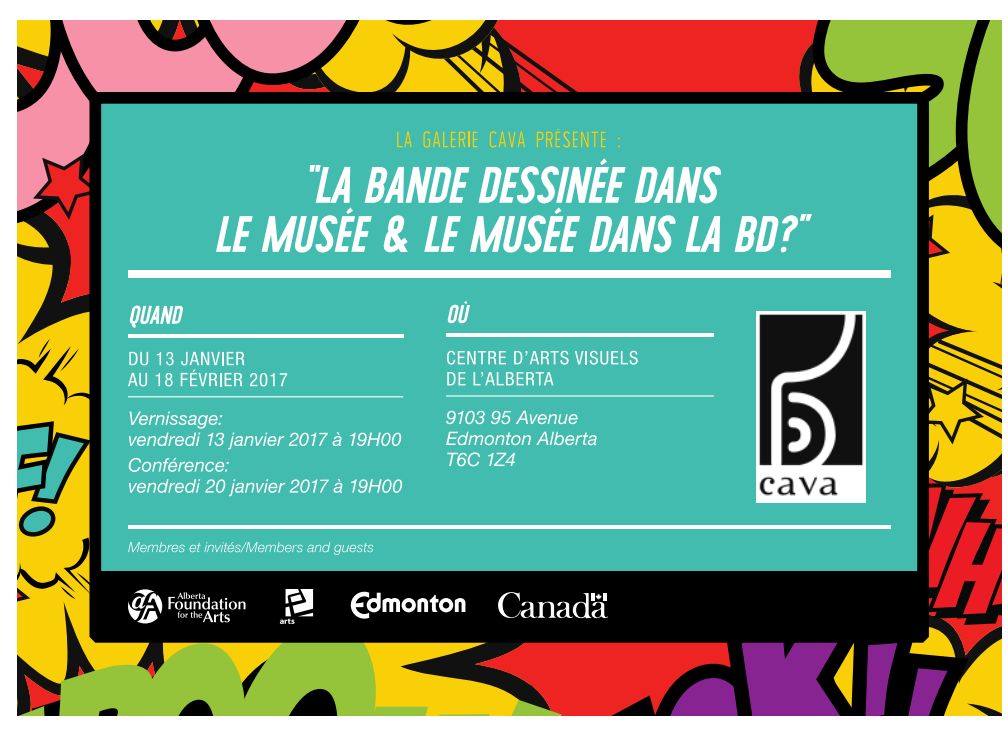

Poster-invitation pour l'exposition à la galerie CAVA, Edmonton, qui a suivi l'exposition qui s'est tenue dans l'espace Rutherford sur le campus de l'Université de l'Alberta.

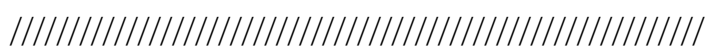

Le jour de la date-limite, nous étions plutôt satisfaits. Car si nous avions reçu seulement une vingtaine de propositions (dont certaines farfelues et sans relation directe avec le sujet), la plupart de ces propositions venaient de partout dans le monde et beaucoup étaient vraiment intéressantes. Nous avons donc sélectionné ce que nous pensions être les dix meilleures 
propositions. Deux mois plus tard nous les avons rassemblées et organisées en fonction de l'espace que nous avions. 
2. Plan de l'espace de l'exposition «Comics-Medium in the Museum », Rutherford South, Rutherford Library, UofA, 20 septembre - 3 octobre 2016.

\begin{tabular}{|c|c|c|c|c|c|c|}
\hline $\mathbf{W}$ & Wall /Mur & Mur & Entrance/Entrée & Mur & Mur & $\mathbf{W}$ \\
\hline $\mathbf{W} \quad \mathbf{P}$ & Panel/Panneau & Panneau & & Panneau & Panneau & $\mathbf{P} \quad \mathbf{W}$ \\
\hline $\mathbf{A} \quad \mathbf{A}$ & Comics in museums & & & & Museums in comics & $\begin{array}{ll}\mathbf{A} & \mathbf{A}\end{array}$ \\
\hline $\begin{array}{ll}\mathbf{L} & \mathbf{N} \\
& \mathbf{N}\end{array}$ & $\begin{array}{l}\text { 1.Belgium, LLN } \\
\text { 2. Belgium, Bxl } \\
\text { 3. France, } \\
\text { Angoulème }\end{array}$ & $\begin{array}{l}\text { Cabinet } \\
\text { Museée } \\
\text { imaginaire } \\
\text { (A moitié vide) }\end{array}$ & & $\begin{array}{l}\text { Cabinet } \\
\text { Albums/books } \\
\text { Tintin-Alph-Art } \\
\text { + Orsay-Olympia }\end{array}$ & $\begin{array}{l}\text {-The Yellow kid } \\
\text {-Crumb's Etudes } \\
\text {-Hergé } \\
\text {-Meurisse }\end{array}$ & $\mathbf{N} \quad \mathbf{L}$ \\
\hline $\mathbf{L} \quad \mathbf{E}$ & $\begin{array}{l}\text { 4. Ohio } \\
\text { 5. NY }\end{array}$ & & & & $\begin{array}{l}\text {-Calvin\&Hobbes } \\
\text {-Astérix-Breughel }\end{array}$ & $\begin{array}{ll}\mathbf{E} & \mathbf{L} \\
& \end{array}$ \\
\hline $\begin{array}{ll}\mathbf{S} & \mathbf{A} \\
& \mathbf{U}\end{array}$ & $\begin{array}{l}\text { 6. Kyoto } \\
\text { 7. Osamu Tezuka } \\
\text { 8. Québec }\end{array}$ & $\begin{array}{l}\text { Cabinet } \\
\text { Chris Ware's } \\
\text { Building Stories }\end{array}$ & & $\begin{array}{l}\text { Cabinet } \\
\text { Louvre } \\
\text {-Mathieu } \\
\text {-Taniguchi }\end{array}$ & $\begin{array}{l}\text {-Desombres } \\
\text {-The GNBCC } \\
\text {-Les Sous-sols } \\
\text {-Les Gardiens }\end{array}$ & $\begin{array}{ll}\mathbf{L} & \mathbf{S}\end{array}$ \\
\hline Door & & & & & & Porte \\
\hline & $\begin{array}{l}\text { Euvres créatives } \\
\text { Pop-up book }\end{array}$ & & & & $\begin{array}{l}\text { Comics/Fashion } \\
\text { Show }\end{array}$ & \\
\hline & $\begin{array}{l}\text { "Autoportrait" } \\
\text { (Alberta) }\end{array}$ & & & & Comics & \\
\hline & $\begin{array}{l}\text { "GhostCat } \\
\text { Virtual" } \\
\text { (Alberta) }\end{array}$ & & & & Fashion & \\
\hline & $\begin{array}{l}\text { "Ears-Eyes" } \\
\text { (Peru) }\end{array}$ & & & & Show & \\
\hline & & $\begin{array}{l}\text { "Table des } \\
\text { opérations" } \\
\text { (France) }\end{array}$ & & $\begin{array}{l}\text { Slobod's } \\
\text { "Make your own" } \\
\text { (Alberta) }\end{array}$ & & \\
\hline Esca- & & Pilier & & Pilier & & Esca- \\
\hline -lier & & & & $\begin{array}{l}\text { "Favela Comics" } \\
\text { (Brazil) }\end{array}$ & & -lier \\
\hline \multirow[t]{2}{*}{ Porte } & & Pilier & & Pilier & & Porte \\
\hline & & Fauteuils & & Fauteuils & & \\
\hline Porte & & $\begin{array}{l}\text { Ecran } \\
\text { "LesBeauxJours" } \\
\text { (Québec) } \\
\text {-"DeathPixel" } \\
\text { (Mexico) }\end{array}$ & $\begin{array}{l}\text { Petit écran } \\
\text { Porte barrée }\end{array}$ & $\begin{array}{l}\text { Ecran } \\
\text {-"Worms" } \\
\text { (Alberta) } \\
\text {-"Comic Splits" } \\
\text { (France) }\end{array}$ & & Porte \\
\hline
\end{tabular}

\section{3b. Explication du plan}

Nous avons donc un long corridor qui est aussi un lieu de passage, en fait, assez fréquenté toute la journée puisque les portes et escaliers permettent de se rendre aux bureaux des bibliothécaires ou 
aux étages supérieurs dans des salles publiques de travail.

L'exposition comprend 3 parties réparties en 3 espaces.

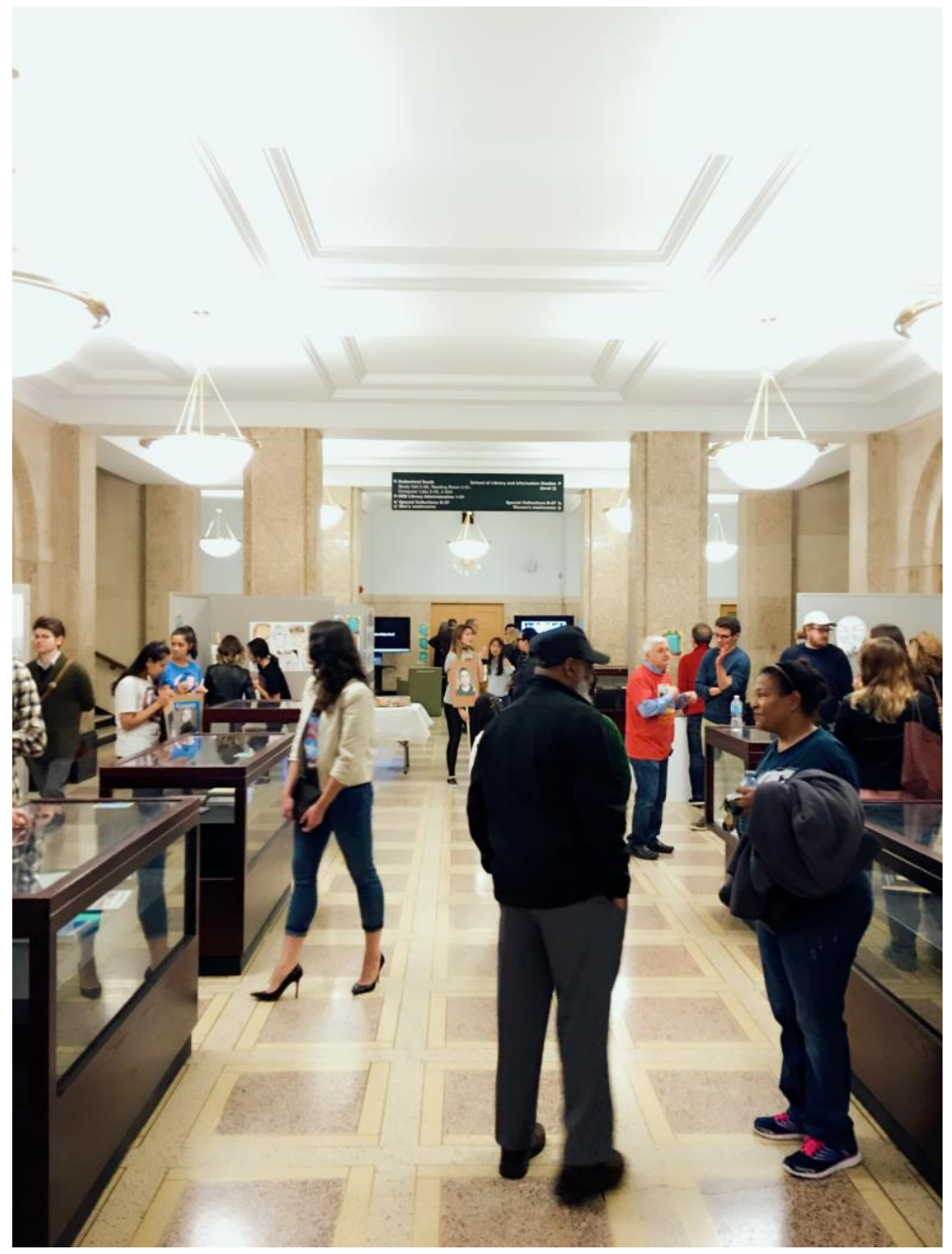

Vue longitudinale de l'espace Exposition de la librairie Rutherford de l'Université de l'Alberta le jour du vernissage vu de l'entrée

A l'entrée à droite (voir le plan à la page précédente), dans la première partie, nous avions une exposition assez traditionnelle, que l'on pourra appeler documentaire, intitulée « la BD au musée » qui montre dix musées de la BD venant des quatre grandes cultures de la BD (les EtatsUnis, le Japon, la France et la Belgique), auxquelles ont été ajouté deux documents (un poster et un dépliant) sur une exposition sur la BD québécoise qui s’est tenue au Québec en 1996. Car même si la BD canadienne n'est pas une des grandes cultures de la BD, nous étions au Canada où il n'y a pas de musée, sauf imaginaire comme représenté à gauche dans une $\mathrm{BD}$ de Seth intitulée Le 
GNBCC (The Great Northern Brotherhood of Canadian Cartoonists, 2011, La confrérie des cartoonistes du Grand Nord, 2012).

A gauche donc (voir le plan), « Le musée dans les BD » montre des images venant de BD dont l'histoire se passe au musée. Celles-ci viennent des cinq mêmes cultures bédéistes (EtatsUnis, Japon, France, Belgique et Canada). Elles se présentent sous forme de posters A3 [29x42cm] épinglés sur des panneaux d'affichage. Dans le cas où les droits n’ont pas été obtenus (e.g. Tintin et l'Alph-Art), le poster est remplacé par un livre qui est exposé dans le meuble-vitrine faisant face aux panneaux.

Nous avions aussi quatre meubles-vitrines [150 x 50cm] disposés face aux panneaux dans lesquels nous avions quelques livres en rapport avec les deux parties documentaires. Dans un premier meuble-vitrine (à droite en entrant), nous avions un seul livre exposé, intitulé Les musées imaginaires de la bande dessinée (Thierry Groensteen et Alii, Ed. de l'An 2, 2004) ; le reste était vide pour justement laisser le «spectaCteur» jouer de son imagination. Dans l'autre de ces meubles-vitrines qui se trouvait face aux posters-photos des musées (à droite en entrant), nous avions placé l'une des BD des plus originales jamais créées qui s'intitule Building Stories (Pantheon, 2012) de l'Américain Chris Ware. Le titre anglais renvoie à la fois à trois significations : histoire d'un bâtiment, construire des histoires et construire des étages («storeys » en anglais britannique). Nous avons donc un livre qui construit des histoires d'étages de bâtiment, établissant un parallèle entre l'architecture, la vie de personnages et la BD. Une coupe transversale d'un tel bâtiment montrerait une série de pièces d'appartement qui ressembleraient ainsi à des cases sur une page. Le livre raconte en effet l'histoire de divers personnages vivant dans une maison à trois étages, un peu à la manière de La vie, mode d'emploi (1978) de George Perec ou de deux livres de Will Eisner, A Contract with God and Other Tenement Stories (1978), traduit en français (Un contrat avec dieu et autres histoires d'immeubles) et publié plusieurs fois sous divers titres et formats ; voir entre autres Le contrat (1993) et The Building (1987) ; L'immeuble, traduit dans New York trilogie, Delcourt (1989). Mais ce livre est aussi une sorte de jeu ou puzzle comportant divers types de documents à lire de diverses manières. Il contenait précisément quatorze documents, dont un album BD classique, divers suppléments de seize pages (comme les comic books), mais aussi un dépliant horizontal en accordéon, et un plateau cartonné articulé en quatre volets. Nous avions donc exposé les diverses parties de ce livre dans la vitrine comme si c'était un «building » dans ses trois dimensions. Ce type de livre-jeu fait aussi référence à toute 
l'histoire de la BD et à ses modes de lecture : de l'album au comic book (équivalent des magazines comme Pilote), aux activités ludiques proposées dans ces magazines (découper des figures, etc). Il y a toutefois une différence essentielle qui est le sujet « sérieux », car celui-ci est une sorte de biographie à la fois triste (histoire, ton) et, paradoxalement, positive, encourageante, puisque le lectaCteur est supposé être actif en co- ou re-construisant l'histoire.

La partie finale et centrale (voir plan) comprend dix œuvres sélectionnées pour une exposition et une œuvre ajoutée après la première sélection. Ces œuvres proviennent de cinq pays différents (France, Pérou, Mexique, Brésil et Canada - Alberta et Québec). Elles sont toutes bien différentes les unes des autres mais ont en commun de défier la manière traditionnelle d'exposer une BD tout en cherchant aussi à étendre et approfondir le monde de la BD et ce qu'est une BD. Un espace spécial était réservé aux quatre vidéos avec deux écrans et deux fauteuils.

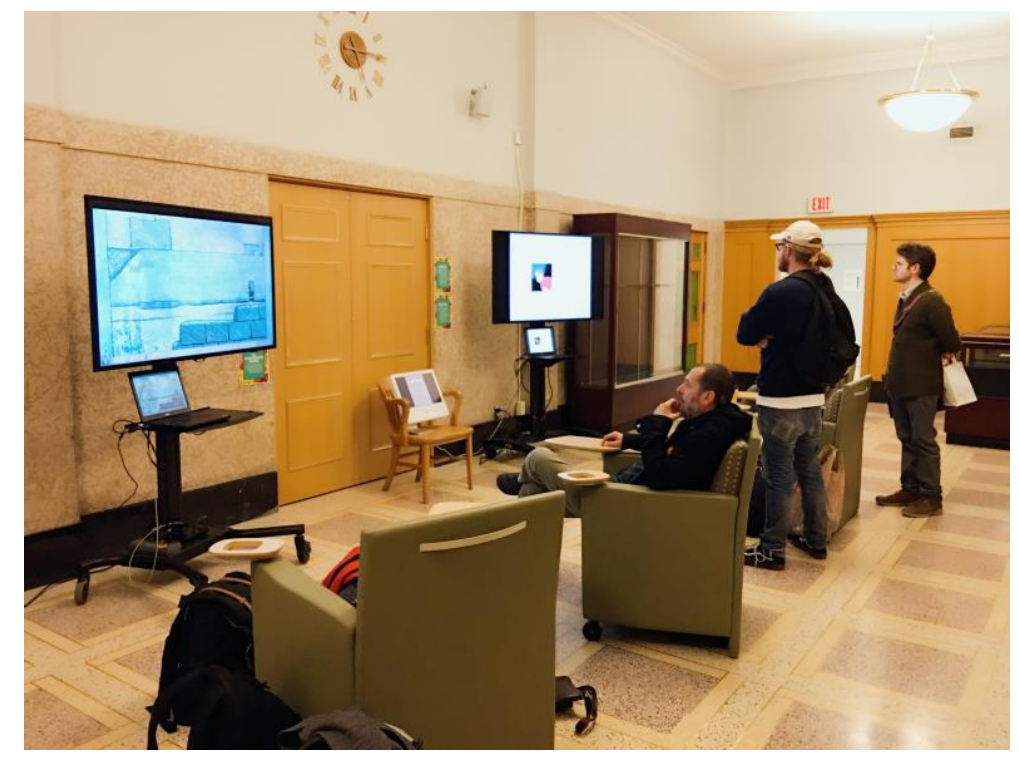

Espace vidéo pour l'exposition dans la bibliothèque Rutherford

Au dernier moment, un fashion-show est venu s'ajouter à l'exposition (en jaune à droite). L'une de nos collègues enseignait en effet un cours sur la mode et l'une de ses classes portait sur un magazine de mode russe, Garage, qui avait sorti un numéro spécial sur la mode, les comics et les superhéros (http://ew.com/article/2016/02/11/mavel-garage-superhero-collaboration/). Ses étudiant/e/s sont venu/e/s défiler lors du vernissage habillé/e/s de t-shirts et costumes de Mickey, Astérix, Superman, Akira et autres personnages de BD/comics/manga. 


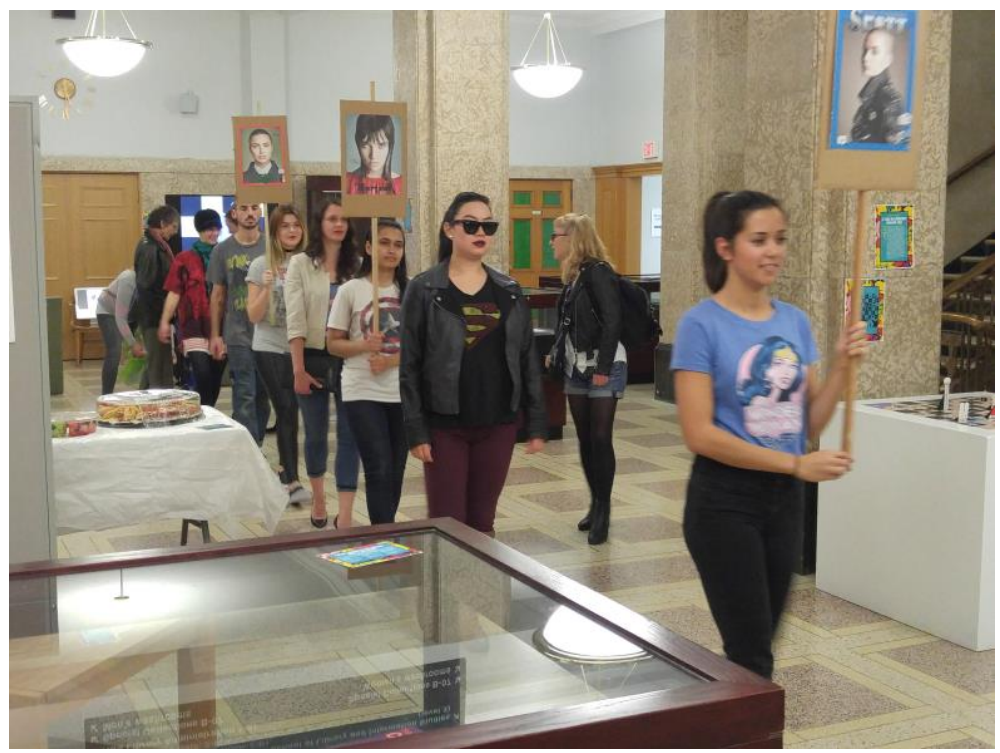

Fashion-show sur les comics dans la mode qui a eu lieu le jour du vernissage de l'exposition à la bibliothèque Rutherford à l'Université de l'Alberta

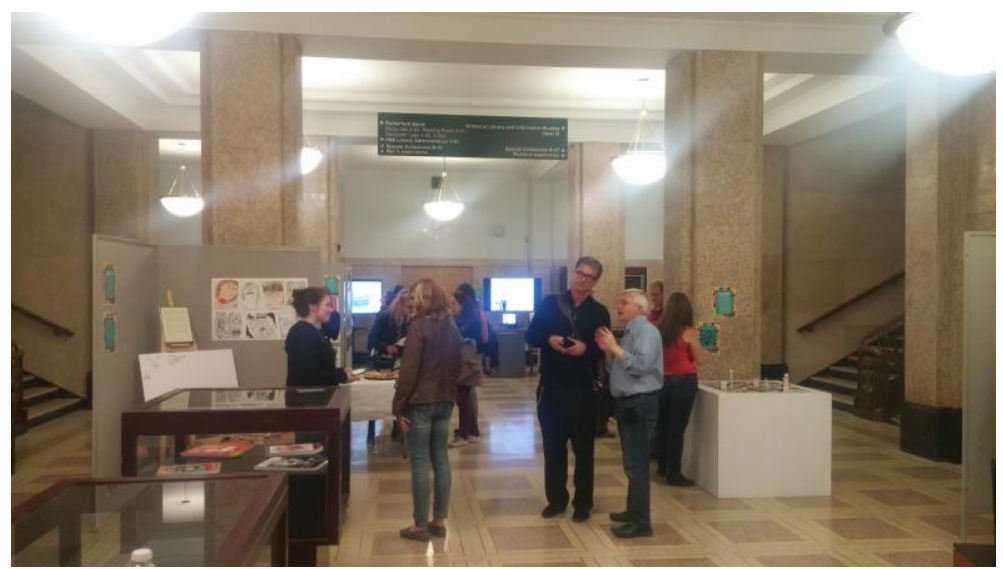

Photo de l'espace Rutherford vu du centre vers le fond où étaient installés deux écrans pour les quatre œuvres vidéo originales 


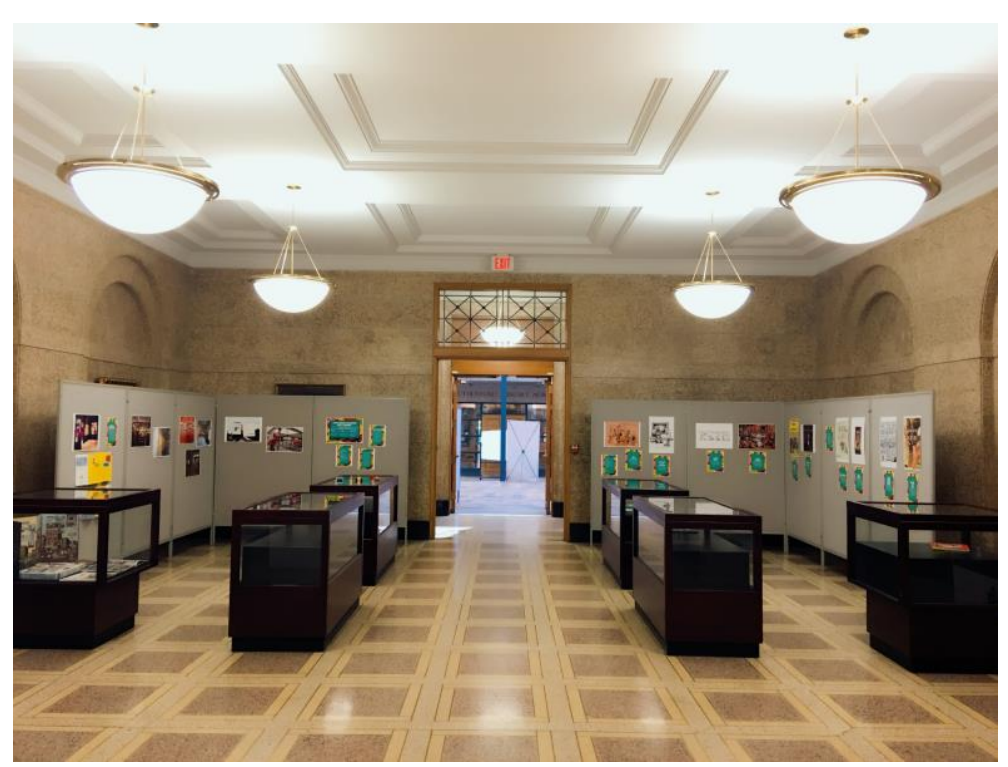

Photo de l'exposition dans l'espace Rutherford vu du centre vers la porte d'entrée

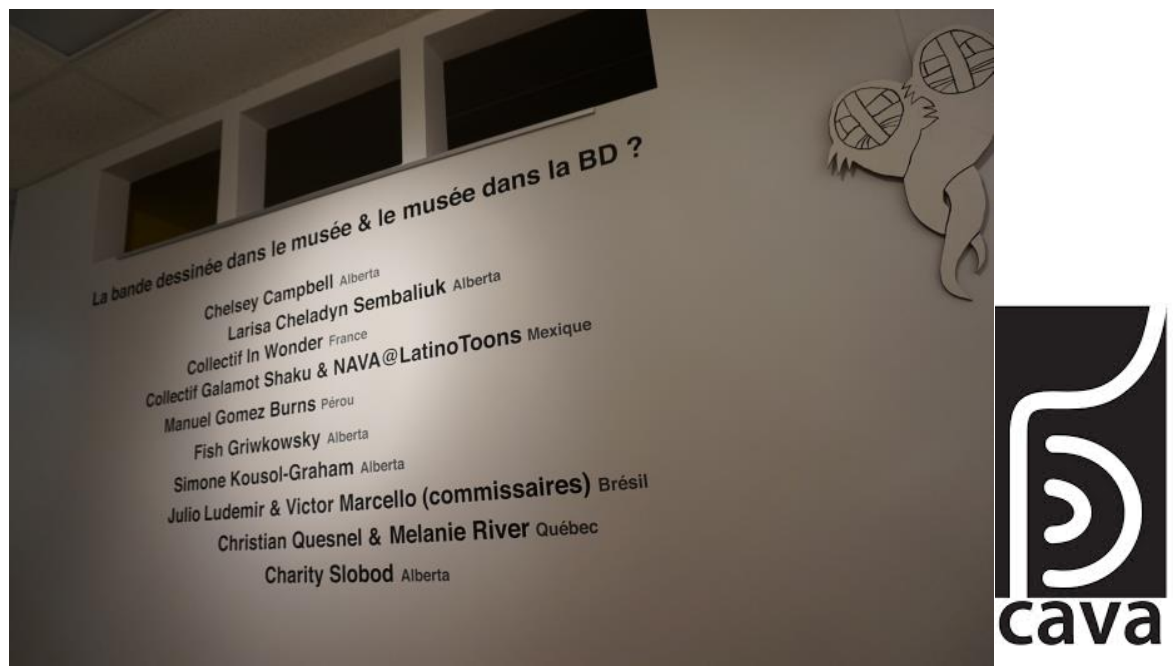

Mur dans l'espace CAVA annonçant le programme de l'exposition.

Logo de la galerie CAVA (Centre d'arts visuels de l'Alberta).

\section{||IIIIIIIIIIIIIIIIIIIIIIIIIIIIIII}

3. Les dix musées sont (accompagnés des images-posters) :

1) Le Musée international du manga de Kyoto, Kyoto, Japon, ouvert en 2006

2) Le Musée du manga d'Osamu Tezuka, Takarazuka, à 30 km de Osaka, Japon, 1994

3) Le Musée Hergé, Louvain-La-Neuve, à $20 \mathrm{~km}$ de Bruxelles, Belgique, 2009 (Architecte : Christian de Portzamparc)

4) Le Musée de la bande dessinée belge, Bruxelles, Belgique, 1989 (Architecte : Victor Horta)

5) Le CIBDI ou la Cité internationale de la bande dessinée et de l'image, Angoulême, France, 2008 
6) Le MoCCA (Museum of Comics \& Cartoon Art [Musée de l'art du comic et du cartoon]), New York, Etats-Unis, 2008

7) Billy Ireland Cartoon Library \& Museum, Columbus, Ohio, Etats-Unis, 1977

8) Exposition au Québec, Canada, 1996

9) «Comics-Medium in the Museum : What a Challenge ! », Rutherford Library, Université de l'Alberta, Edmonton, Canada, 2016

10) «La bédé au musée : un défi », CAVA, Edmonton, Canada, 2017.

|||||||||||||||||||||||||||||||||

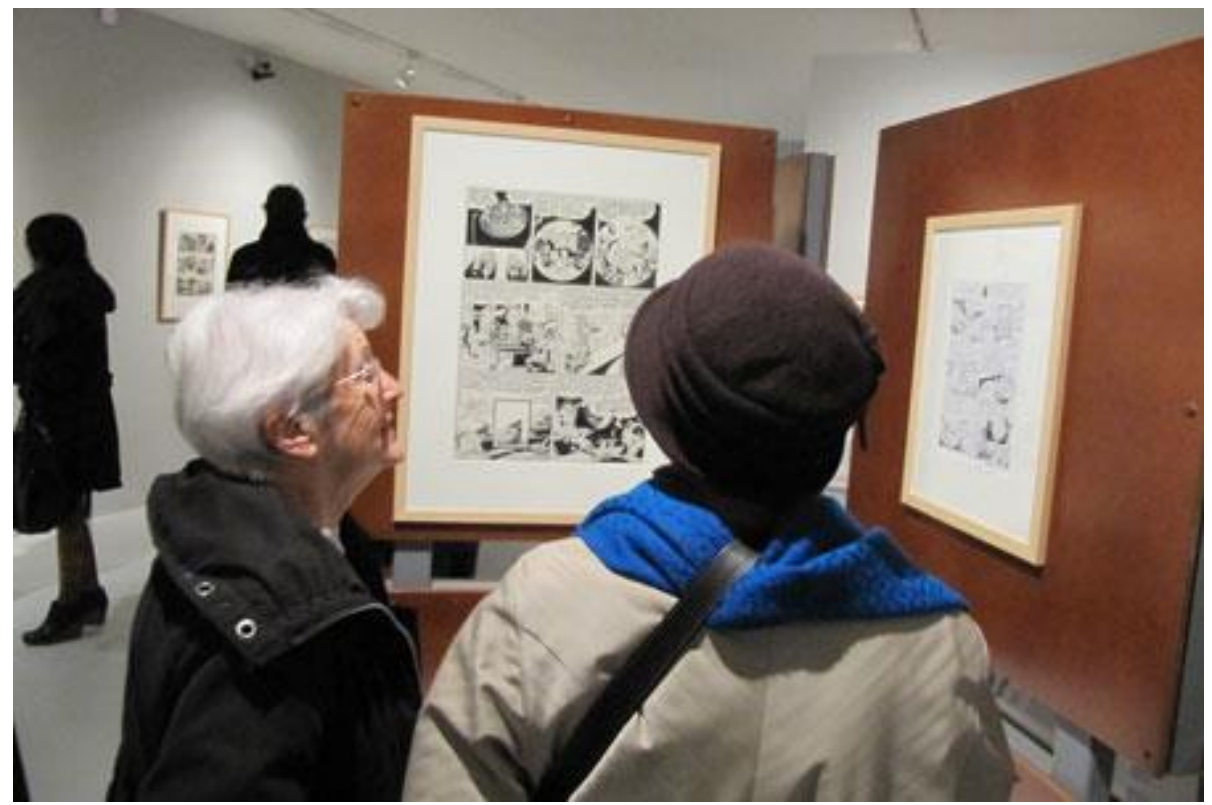

Exposition dans une des salles du CIBDI d'Angoulême

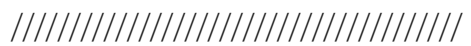

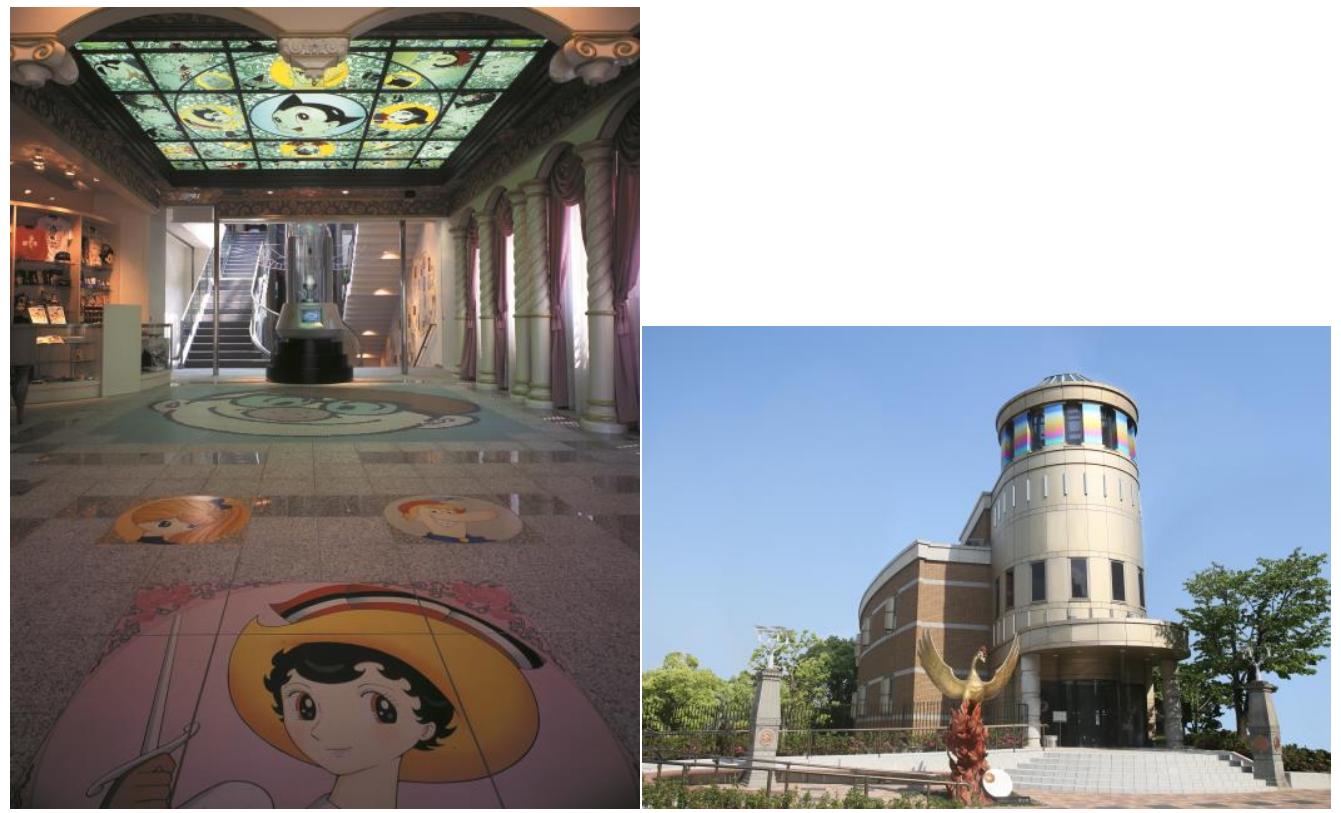

Musée Tezuka Osamu, Takarazuka, Japon ; extérieur et intérieur (Permission Takarazuka City) 


\section{//////////////////////////}

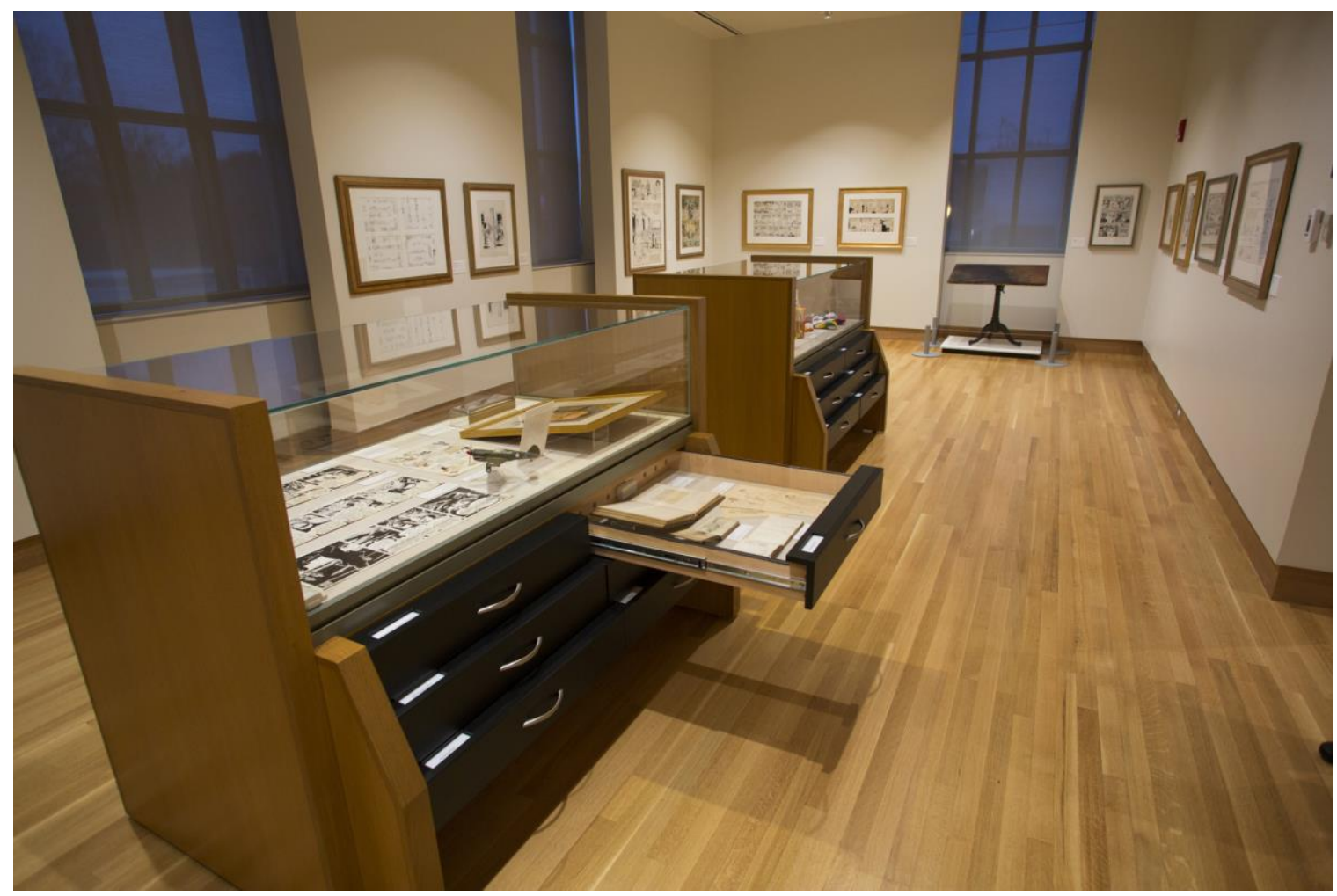

Galerie des tiroirs/Drawers Gallery au musée Billy Ireland Cartoon Library and Museum, Columbus, Ohio, USA. (Permission Treasures Gallery, The Ohio State University Billy Ireland Cartoon Library \& Museum)

\section{|||||||||||||||||||||||||||||||||||||||||||||||||||||||||||||||||||||||||||}

\section{Les dix BD muséographiques sont :}

\section{1) Richard Outcault, The Yellow Kid, Etats-Unis, 1896}

La première image de cette exposition est intitulée « In the Louvre » («Au Louvre ») et sous-titrée «The Yellow Kid takes in the masterpieces of art», (« Le Yellow Kid [1'enfant jaune] assimile les chefs-d'œuvre artistiques »). Elle fait partie d'une série où l'auteur Outcault et ses partenaires emmènent ce jeune garçon « mal léché » autour du monde pour se moquer de diverses hiérarchies élitistes, politiques, sociales, culturelles et artistiques. Elle représente ce qui pourrait être une scène de cauchemar pour les élites newyorkaises fascinées à l'époque par le grand art européen que les Etats-Unis n'avaient pas encore reconnu chez eux. Si vous faites spécialement attention, vous verrez que la célèbre statue de Milo [sans bras] dit : «Si j’avais mes bras je donnerais une fessée à cet enfant ! » 


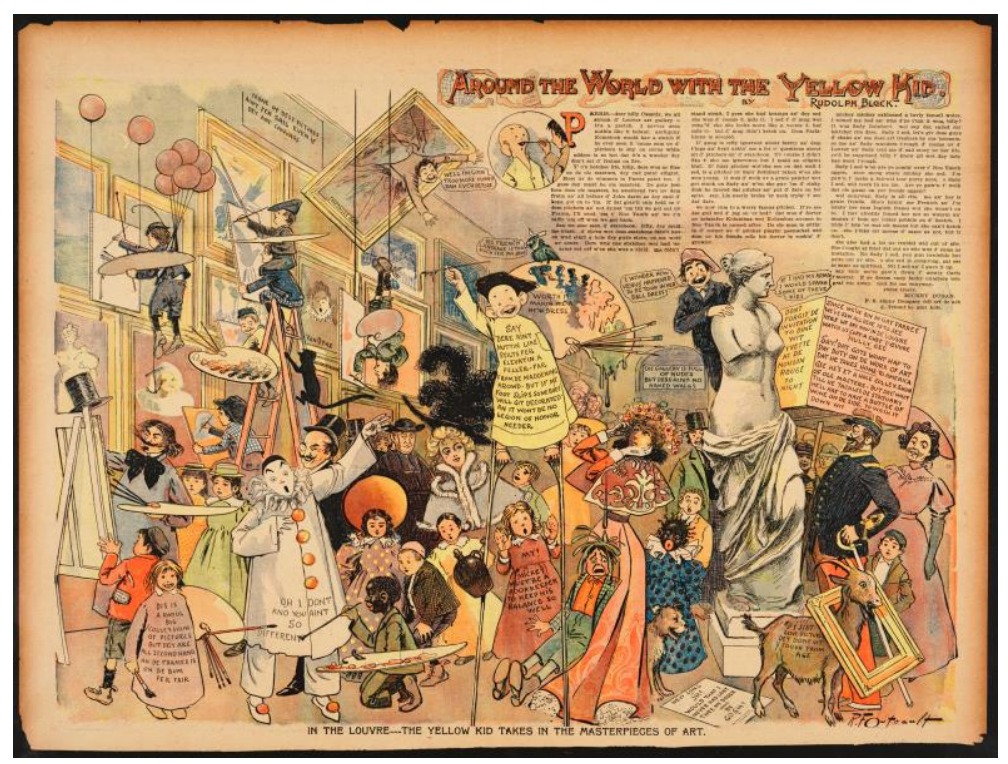

« The Yellow Kid at the Louvre », « The Yellow Kid takes in the masterpieces of art », Richard Outcault, 1896. (Domaine public)

\section{2) Robert Crumb, «'Etudes de figures' au musée d'art », Etats-Unis, années 1970}

Crumb est justement reconnu comme l'un des grands maîtres des comics de l'underground (comix) et de la BD en général. Il fut d'ailleurs l'un des premiers artistes de BD à être exposé dans un musée, d'abord avec d'autres, dans « Masters of American Comics » au Musée juif de NY en 2006 et ensuite, seul, à l'ICA, Institute of Contemporary Art (Institut d'art contemporain) à Philadelphie en 2008. Il est aussi un exemple assez typique du sexisme dans le monde de l'art et des comics/BD. Cette image révèle cependant la difficulté de voir cet artiste comme simplement sexiste, car comme souvent ses images sont à double-entendre (utilisant la mise en abyme ou autres techniques réflexives). Ainsi ici, si les femmes qui regardent la peinture où sont peintes des femmes qui sont assez typiquement des objets sexuels du regard masculin, elles sont aussi elles-mêmes des objets de ce même regard par le personnage masculin.

Le dessin de Robert Crumb intitulé « 'Figure Studies' at the Art Museum » est disponible en ligne : https://www.google.ca/search?q=robert+crumb+figures+studies\&safe=off\&rlz=1C5CHFA_enC $\underline{\text { A691CA691\&source }=\operatorname{lnms} \& \text { tbm=isch\&sa=X\&ved=0ahUKEwiIqIeCjuTWAhXlz1QKHQcmA3 }}$ $\underline{\text { AQ_AUICigB \&biw }=1157 \& \mathrm{bih}=523 \# \text { imgrc }=W E c D \_4 K k S G-Q c M}$ 


\section{3) Bill Watterson, Calvin \& Hobbes, Etats-Unis, 1990}

Les personnages de cette bande/strip sont célèbres dans le monde entier. Nous savons que sous l'apparence d'un pur divertissement, ces strips sont de bons exemples de la formule «apprendre en s'amusant ». Ils explorent avec humour et tendresse des problèmes comme l'instruction publique, l'environnement et autres problèmes sociaux ou philosophiques. Les noms de ces deux personnages sont inspirés par ceux du théologien franco-suisse Jean Calvin et du philosophe anglais Thomas Hobbes. Ce strip-ci est un parmi quatre ou cinq qui interrogent la hiérarchie artistique. Cette image de Calvin and Hobbes de Bill Watterson est disponible en ligne : https://www.google.ca/search?q=bill+watterson+calvin+art\&rlz=1C5CHFA_enCA691CA691\&t

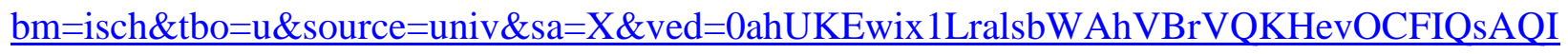
$\underline{\text { Jw\&biw }=960 \& \text { bih }=534 \# \text { imgrc }=W R W 1 \text { ABupMgjEtM }}$

\section{4) Uderzo \& Goscinny, Astérix chez les Belges, France, 1977}

Beaucoup lisent « Astérix » juste pour rire. Et c'est certainement aussi ce pour quoi les auteurs ont créé ces BD historico-comiques. Mais, si parfois ce sont de purs slapsticks, le plus souvent ces gags sont faits pour se moquer des travers humains ou plus spécifiquement français, et des stéréotypes culturels et ethniques. D'autres fois aussi, ces histoires établissent un pont avec la grande culture comme la peinture. Ainsi, chaque aventure d' «Astérix» finit par un repas communal, et ici, puisque cette aventure se passe en Belgique, les auteurs ont choisi comme modèle une peinture célèbre, Le mariage paysan (1567) du peintre «belge » Breughel le Vieux. Cette parodie fait ainsi le pont entre deux arts populaires (la peinture est en effet aussi populaire au double sens de son contenu et de sa popularité), mais séparés par des préjugés «classiques » (grand Art / BD ou grand art / divertissement).

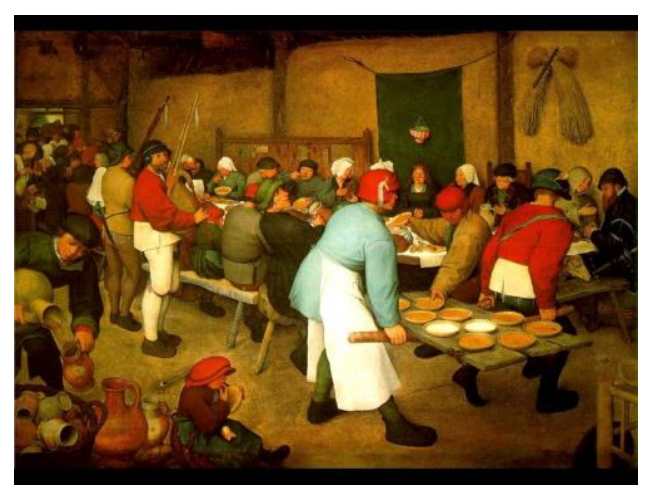

Peinture de Breughel le Vieux, Le mariage paysan, 1567 (Domaine public) 
La page d'Astérix permet la comparaison :

http://www.mage.fst.uha.fr/asterix/allusion/bruegel.html

\section{5) Hergé, Tintin et l'Alph-Art, Belgique, 1986}

Pour diverses raisons qui ne sont pas claires (Etait-il trop occupé par ses invitations comme célébrité ? Etait-il trop malade et/ou était-ce dû à sa difficulté de réconcilier ces deux arts qu'il aimait, BD et peinture ?), Hergé n'a pas fini cette dernière aventure de Tintin. L'album fut néanmoins terminé par plusieurs artistes, mais illégalement, puisque Hergé avait explicitement interdit de continuer son œuvre après sa mort. Malgré de nombreuses références à des objets de valeur dans ses aventures précédentes (en fait, à des objets classés ethnographiques plutôt qu'artistiques), ceci est la première histoire qui se passe entièrement dans les milieux artistiques. Après avoir découvert qu'un marchand d'art a été assassiné, Tintin découvre une conspiration sur des faussaires d'art orchestrée par un gourou religieux (« un faussaire de religion »).

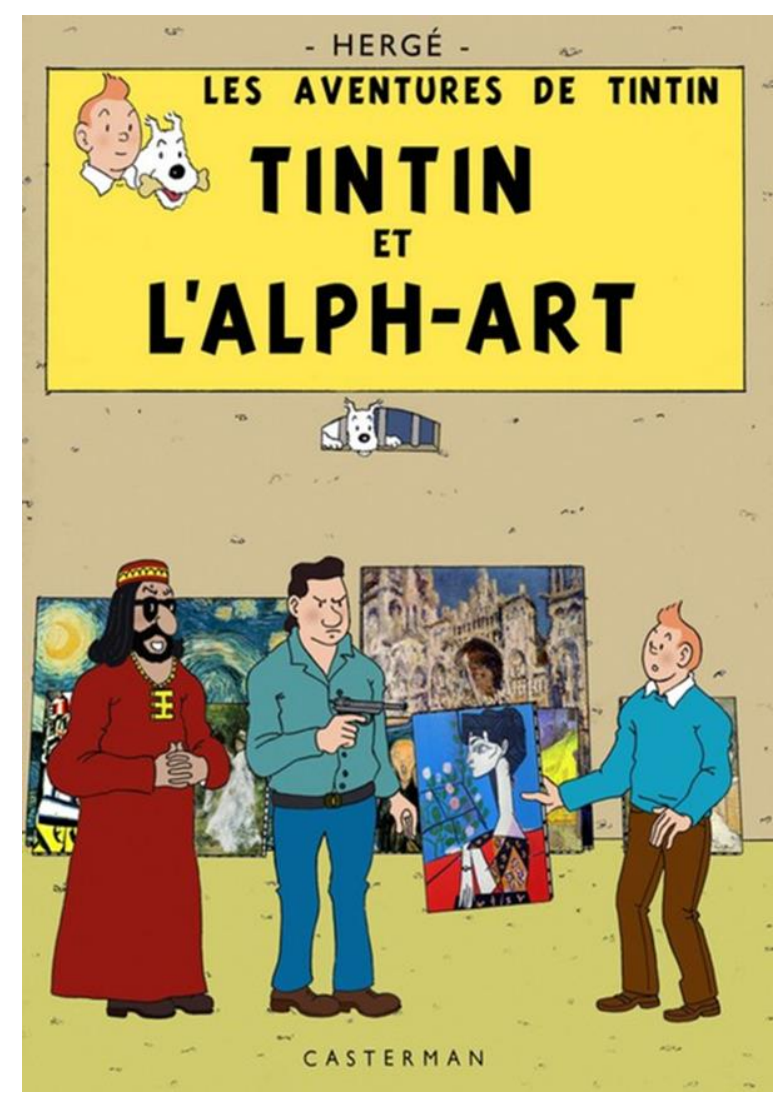

Couverture de l'album d'une des parodies de l'album original inachevé. 


\section{6) Peeters \& Schuiten, L'affaire Desombres, France, 2000}

L'affaire Desombres qui peut aussi se comprendre comme l'affaire des ombres [mystérieuses] est un projet multimédia et transmédial (livre, musée, musical, dvd, etc.) créé pour défier les diverses manières d'aborder la BD et les arts. Il a été produit par deux grands noms de la BD franco-belges, Benoit Peeters et François Schuiten. Il raconte l'histoire d'une femme qui se lance sur les traces d'un peintre du dix-neuvième siècle appelé Augustin Desombres et dont l'œuvre aurait entièrement disparu, sauf pour quelques traces comme des lettres, partitions musicales, etc.

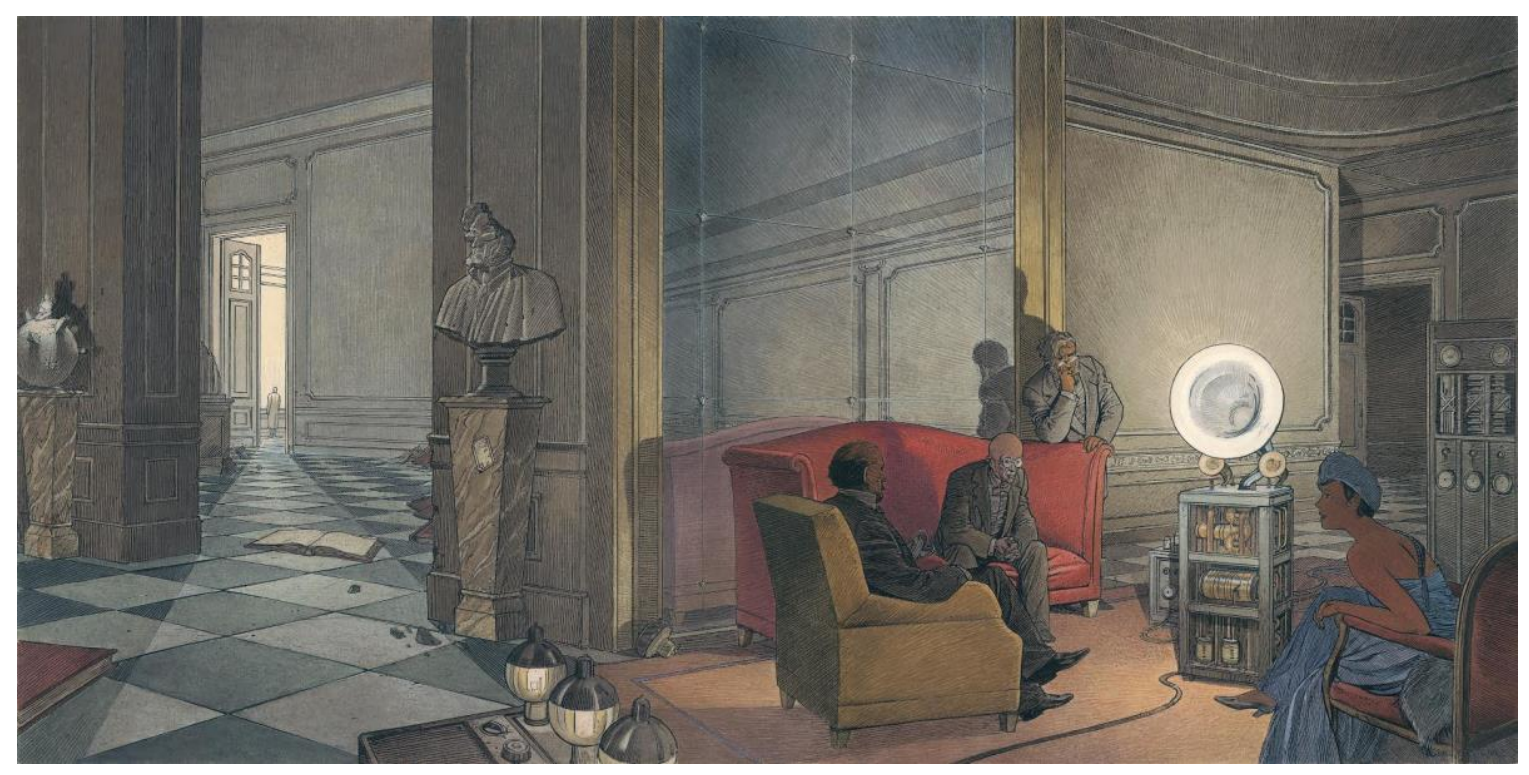

Partie de couverture de l'album Le Musée A. Desombres ( Schuiten-Peeters, Le Musée A. Desombres, 1990. Tous droits réservés.)

\section{7) Marc-Antoine Mathieu, Les sous-sols du révolu, France, 2010}

Le mot révolu dans le titre signifie «ce qui est passé » mais il est aussi l'anagramme du mot «Louvre » et pourrait aussi renvoyer à révolu-tion incomplète. Malgré le format traditionnel du 64cc (soixante-quatre pages Carton Couleur), il raconte une histoire peu conventionnelle et d'une manière non-conventionnelle qui est celle d'un expert chargé de faire l'inventaire d'un musée en 1840. L'auteur, Marc-Antoine Mathieu, est l'un des artistes les plus talentueux de la BD française contemporaine, mettant souvent en images l'autoréflexivité dans l'art et la vie. Ce « roman graphique » fait partie d'une série d'albums sur le Louvre dans la collection « Le Louvre par .... » (voir aussi Taniguchi et Meurisse ci-après), coéditée par les éditions de BD Futuropolis et le Musée du Louvre. 


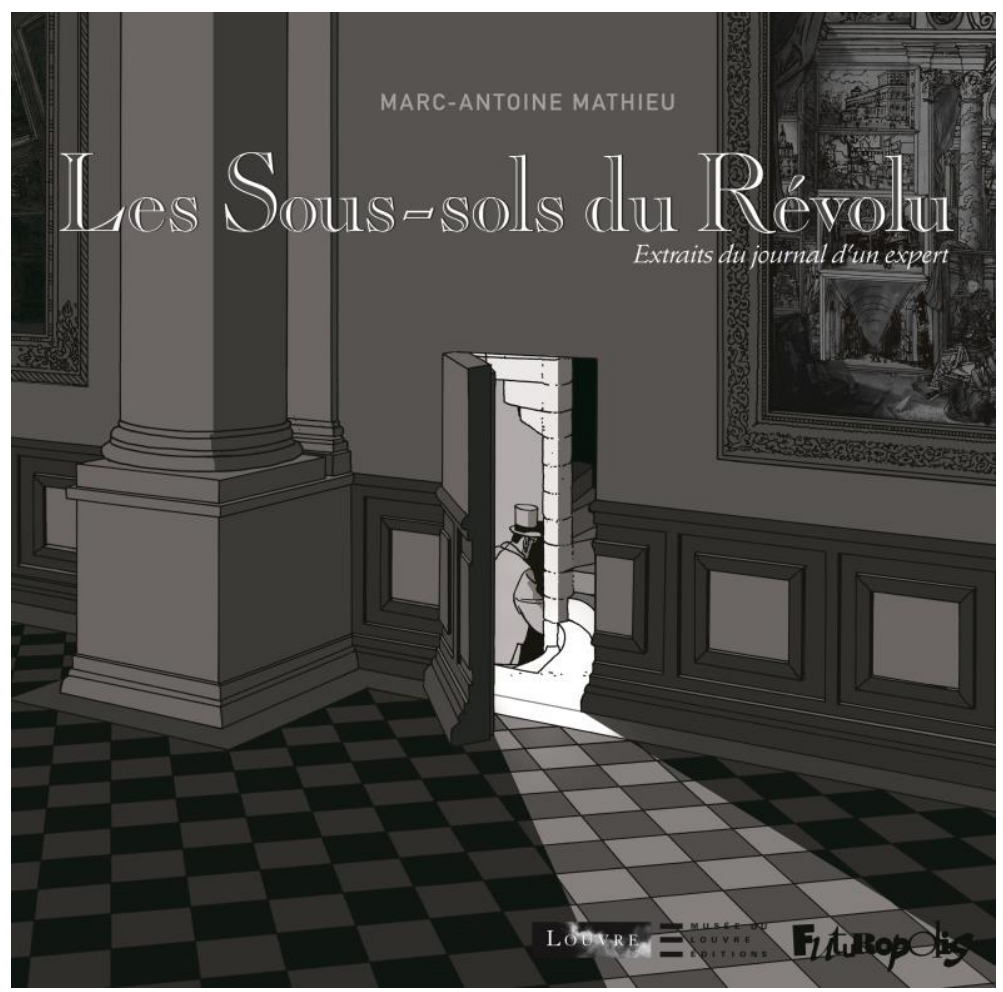

Couverture de l'album Les sous-sols du révolu (Permission MATHIEU@FUTUROPOLIS, 2006)

\section{8) Catherine Meurisse, Moderne Olympia, France, 2014}

Ceci est le seul album de bande dessinée fait par une femme artiste dans cette liste de dix albums, ce qui représente en fait à peu près le pourcentage de femmes bédéistes en France en 2016 (10\%). Mais quelle histoire extraordinaire, amusante et intelligente ! Le titre fait évidemment référence au tableau d'Edouard Manet, Olympia (1863). L'histoire peut se lire comme une critique de la longue tradition sexiste dans les Beaux-Arts et dans la bande dessinée. Elle raconte l'histoire d'une actrice, Olympia, qui refuse de jouer les seconds rôles dans un spectacle et est alors prise entre les deux «clans » artistiques de cette deuxième partie du dix-neuvième siècle : d'une part, le Salon officiel et, de l'autre, le Salon des Refusés. L'album a été coédité par le Musée d'Orsay et les éditions Futuropolis. (Moins d'un an après cet album, Meurisse a échappé au massacre du 7 janvier 2015 de Charlie Hebdo parce qu'elle est arrivée en retard à la réunion !) 


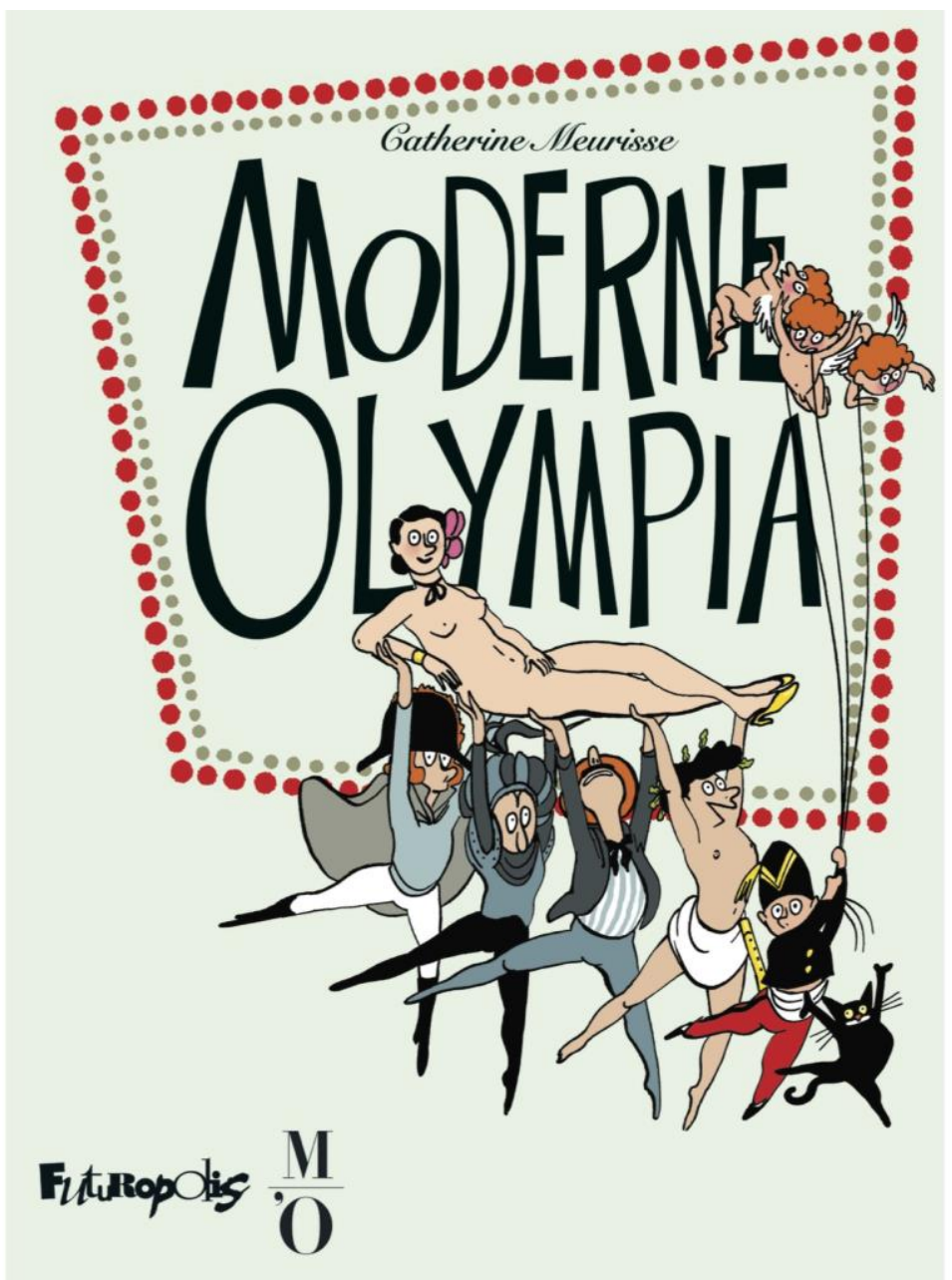

Couverture de l'album Moderne Olympia (Permission MEURISSE@FUTUROPOLIS, 2014)

\section{9) Taniguchi Jiro, Les gardiens du Louvre, Japon, 2010}

Cette image est la couverture de l'album publié dans la collection « Le Louvre par ... » coédité par le Musée du Louvre et l'éditeur de BD Futuropolis. Bien que moins populaire que les mangakas qui ont produit les Dragon Ball et autres Ranma 1/2, Taniguchi est un artiste japonais des plus intéressants. Dans sa «non aventure », on suit un artiste japonais visitant le Louvre et faisant de mystérieuses rencontres comme celles de la Victoire de Samothrace, Corot, Van Gogh, Asai Chu, etc. Le livre est un grand format $(20 \times 28 \mathrm{~cm})$, utilise la couleur directe et se lit dans le sens japonais (de droite à gauche). 


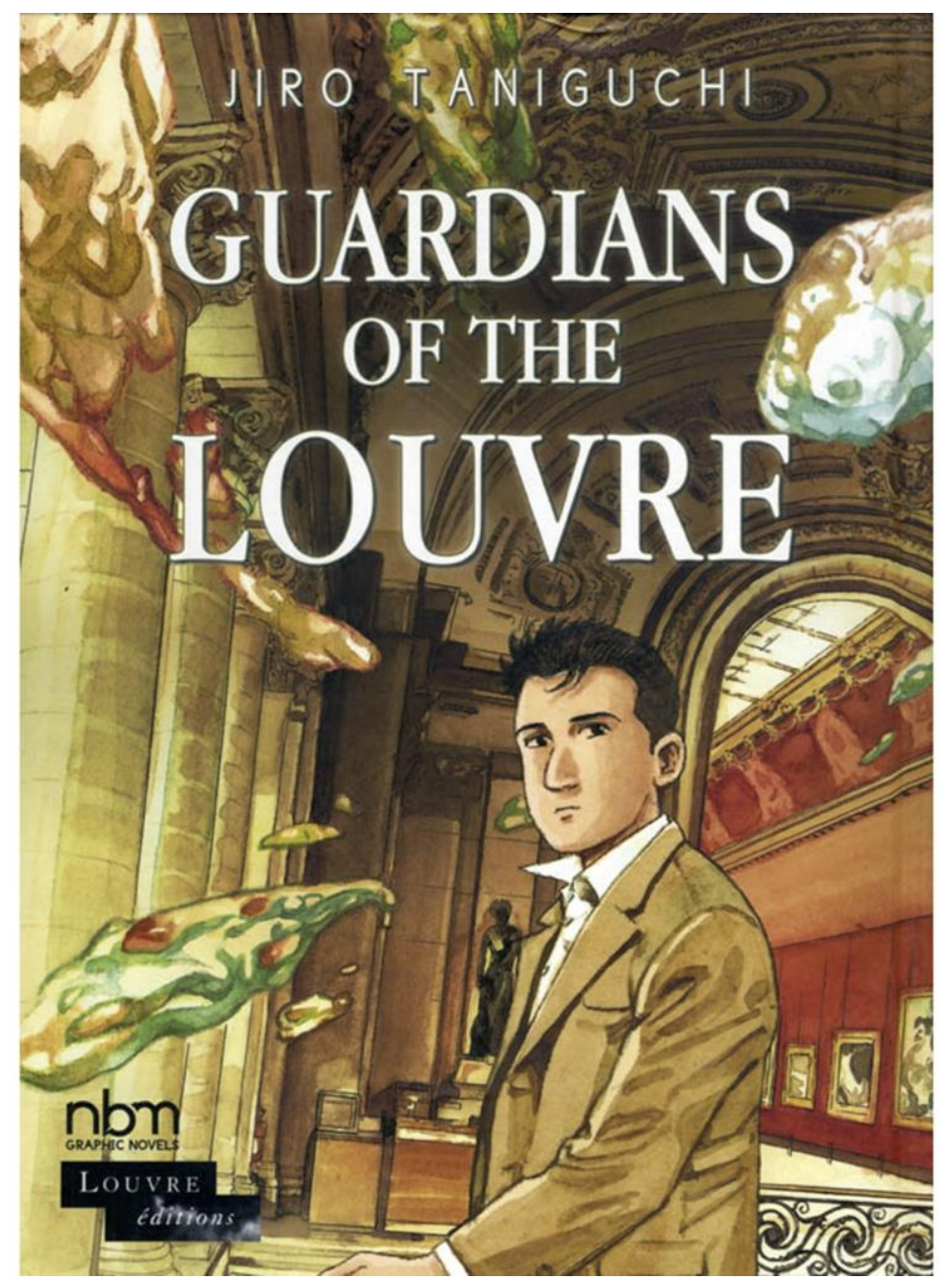

Couverture de l'album Les gardiens du Louvre (Permission TANIGUCHICFUTUROPOLIS, 2014)

10) Seth, The Great Northern Brotherhood of Canadian Cartoonists, 2011, Canada [Aka The GNBCC) ; La confrérie des cartoonistes du Grand Nord, 2012]

Ce long titre est aussi connu comme le GNBCC. L'album, publié par le montréalais Drawn \& Quarterly, a été créé par le Canadien Seth. Il raconte une histoire alternative du Canada. Seth emmène les lecteurs dans un tour du bâtiment de la GNBCC situé à Dominion, une ville fictive dans l'Ontario. Il nous donne une vue générale des artistes canadiens de BD, mentionnant de vrais auteurs comme Doug Wright (aujourd'hui le nom d'un des prix de la BD canadienne) mais aussi des noms fictifs. S’y mêlent nostalgie et humour de manière créative. 


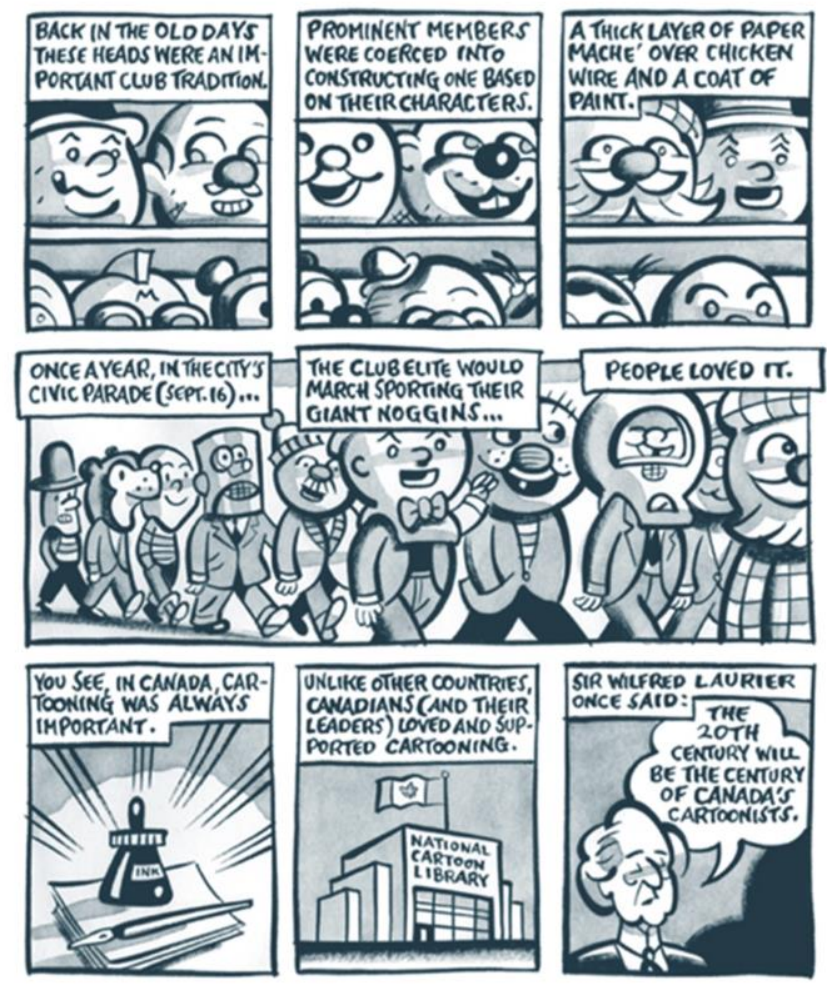

Page 6 de l'album The GNBCC (Permission gracieuse de Seth)

\section{Les œuvres créatives sont :}

Les musées ont commencé à penser à exposer des bandes dessinées (BD ou bédé) quand le mouvement de reconnaissance de la $\mathrm{BD}$ comme neuvième art a commencé à s'épanouir dans les années 1980. Les musées avaient aussi besoin de nouveaux clients car, avec de rares exceptions comme des événements extraordinaires telles que les expositions d'artistes-stars comme Van Gogh, Picasso ou les Impressionnistes, et malgré d'énormes campagnes de marketing, ils étaient de moins en moins efficaces pour attirer des foules importantes et plus jeunes. En même temps, de plus en plus de BD commençaient à être de meilleures qualités, visant des publics de plus en plus adultes et attirant des artistes de plus en plus talentueux. Enfin, les éditeurs acceptaient d'investir de plus en plus d'argent pour employer, par exemple, une meilleure qualité de papier et des formats de plus en plus variés et adaptés aux exigences de l'œuvre. C'est alors que les musées ont commencé à penser que les BD pourraient être acceptables, profitables et même intéressantes. D’autre part, les artistes de BD étaient devenus suspicieux vis-à-vis des « grands arts » comme la peinture puisqu'ils avaient été si longtemps méprisés ou avaient été réappropriés par des artistes 
comme Roy Lichtenstein de manière quelque peu condescendante. Certains pensaient ou disaient même qu'ils se débrouillaient très bien en vivant et en créant en-dessous des radars officiels des élites artistiques. De plus, les BD ont été reconnues d'abord et surtout par les communautés littéraires (lecteurs, académiques, etc.] et, ensuite, comme l'a très bien montré Bart Beaty dans Comics vs Art (2012), par les gardiens des portes des institutions artistiques.

Le processus d'acceptation mutuelle a donc été lent mais continu depuis les années 1980, spécialement dans les trois grandes traditions de la BD, des Comics et du manga. Cependant, montrer des BD dans un musée reste encore un défi pour d'autres raisons. Une BD est un média très différent d'un musée. Les $\mathrm{BD}$ comme les romans se lisent, la plupart du temps, seul et en privé ; les dimensions du livre et plus encore des cadres et de ce qu'il y a à l'intérieur impliquent une distance proche et une isolation relative par rapport à l'extérieur (lecture mentale).

Un musée est habituellement un espace couvert qui vous entoure et que vous visitez pour admirer une œuvre d'art sur un mur ou un piédestal, et à une certaine distance à cause de son caractère unique et quasiment «sacré », habituellement entouré d'autres spectateurs-visiteurs.

Comment donc réconcilier les approches de ces deux médias?

Comment montrer une BD qui demande une lecture privée individuelle sécularisée et une lecture muséale qui est collective, publique, et quasiment sacrée ?

Aujourd'hui dans un mode où la séparation entre le privé et le public s'efface, où le transmédia est devenu plus commun que la pureté moderniste d'un art (voir Clément Greenberg) et où les gens demandent plus d'interactivité, les œuvres présentées ici offrent certaines solutions.

La partie finale et centrale comprend dix œuvres sélectionnées pour une exposition et une œuvre ajoutée après la première sélection. Ces œuvres proviennent de cinq pays différents (France, Pérou, Mexique, Brésil et Canada - Alberta et Québec). Elles sont toutes bien différentes les unes des autres mais ont en commun de défier la manière traditionnelle d'exposer une $\mathrm{BD}$ tout en cherchant aussi à étendre et approfondir le monde de la BD et ce qu'est une BD.

\section{6b. Les dix artistes ou groupes d'artistes exposant sont par ordre alphabétique :}

1) Campbell, Chelsey ; Alberta

2) Cheladyn Sembaliuk, Larisa ; Alberta

3) Collectif In Wonder ; France

4) Collectif Galamot Shaku \& NAVA ; Mexique 


\section{5) Gomez Burns, Manuel ; Pérou \\ 6) Griwkowsky, Fish ; Alberta \\ 7) Kousol-Graham, Simone ; Alberta \\ 8) Ludemir, Julio \& Victor Marcello ; Brésil \\ 9) Quesnel, Christian, \& Mélanie River ; Québec \\ 10) Slobod, Charity ; Alberta}

Ci-après, vous trouverez les dix textes qui accompagnaient les œuvres. D’abord écrits ou traduits en anglais, ils étaient imprimés sur un poster placé juste à côté de chaque œuvre.

Les dix œuvres représentent chacune un défi à la présentation de la BD en général et dans l'espace muséal en particulier.

Ainsi la plus conventionnelle est-elle sans doute celle de Manuel Gómez Burns (Pérou, 1) qui a créé une BD en six panneaux qui pourraient être une aventure publiée dans un magazine de BD. Cependant, l'intérêt de ce long strip intitulé Onomatopoeia anthropomorphica vient de ce jeu avec les sens (les yeux et les onomatopées) mis en images. En effet, si les yeux renvoient directement au regard, sens unique pour apprécier la BD, les onomatopées (éléments qui, pour beaucoup, définissent par excellence la BD) renvoient au son qui ne peut être que visuel/visualisé dans la $\mathrm{BD}^{1}$.

Pop-up' Culture (Alberta, 2) présente une BD faite sur un pop-up rétractable de deux mètres de haut sur lequel l'artiste Larisa Sembaliuk Cheladyn a dessiné une adaptation de la nouvelle «Boys \& Girls » («Garçons et filles ») de l'écrivaine Alice Munro, prix Nobel 2013. Ce pop-up, fait au départ pour une classe sur la BD canadienne enseignée par Chris ReynsChikuma à l'Université de l'Alberta, défie le moyen traditionnel de présenter une BD (sous forme de page affichée au mur comme un poster), d'autant plus que c'est une adaptation d'une « grande » œuvre littéraire.

Le projet de Julio Ludemir et Victor Manuelo (Brésil, 3) ironiquement intitulé No Title est apparemment aussi une BD traditionnelle mais les conditions de production et de distribution sont assez différentes. En effet, ils ont mis en contact des habitants de favelas pour raconter leurs histoires personnelles, familiales et locales, et cinq bédéistes locaux pour les «bandedessiner », pour ensuite les afficher comme grands posters sur les murs des deux quartiers de favelas concernés.

L'autoportrait (Alberta, 4) de Chelsea Campbell est une autre manière apparemment conventionnelle pour un musée puisque c'est un dessin encadré. Mais le dessin est une œuvre 
originale et utilise le format du cadre traditionnel pictural (une seule case) et certaines conventions du monde des comics (la caricature, le dessin éditorial, etc.) pour critiquer l'ambivalence dans laquelle le consommateur (albertain, canadien, etc.) est pris.

Avec les deux œuvres produites par le collectif français In Wonder, on s'éloigne de plus en plus de la BD traditionnelle. Le premier projet, La table des opérations (France, 4), est effectivement une table-échiquier sur laquelle peuvent s'effectuer des opérations de déplacement d'une dizaine de figures qui peuvent être des personnages, des objets ou des signes dans l'espace tridimensionnel à partir des cases. C'est une œuvre qui défie la convention des deux dimensions de la BD. Elle se rapproche à la fois des oubapiens et des « building stories » de Chris Ware (voir ci-dessus) et des groupes éditeurs et expérimentateurs comme Fréon-Amok-Frémok.

La deuxième de leurs œuvres est une vidéo (France, 5) intitulée Comic Splits qui reprend certains éléments de La table des opérations comme la table-jeu d'échec mais cette fois présentée en « dessin animé » pour établir des connections entre la BD et le dessin animé, basées à la fois sur la linéarité (où une séquence de lecture est imposée) et l'association libre.

De la même manière, dans Death by Pixel (mort par pixel), le collectif Galamot Shaku \& Nava@ LatinoToons (Mexique, 6) présente une vidéo basée sur des traditions visuelles très diverses, des plus anciennes telles que les arts amérindiens et le textile mésoaméricain, aux plus modernes comme le comix underground et le jeu vidéo 8 bits, incluant les plus avancées en 2016 comme le gif-art (le Graphic Interchange Format-format d'échange d'images) et la VJ-ing (VidéoJockey), produisant une œuvre bédéiste animée d'avant-garde engageante sur la mise à mort.

Similairement, Christian Quesnel, en collaboration avec Mélanie River et Evelyn Accad (écrivaine libanaise) dans Oh Les beaux jours (Québec, 7), nous présente un bref récit sous la forme d'une BD multimédia ajoutant à la fois le son (la voix et le bruit du vent) et quelques mouvements de caméra, pour mettre en question les différentes formes que peut prendre la bande dessinée à l'extérieur du livre standard imprimé et notamment dans la mise en exposition de celleci. Outre le son, l'espace inter-iconique est ici transposé en coupes progressives d'une image à l'autre, ajoutant volontairement un aspect vaporeux au récit et tentant de préserver cet espace où le lecteur effectue le lien mental entre les images. Cette démarche est aussi l'occasion pour Quesnel d'apprivoiser un récit dont la case est uniforme.

De même, Simone Kousol-Graham, dans Worms (Alberta, 8), utilise divers styles d'images, fixes et animées, son et texte parlé pour transmettre des informations que l'on pourrait 
qualifier d'empathiques sur les vers de terre.

Journaliste et critique d'art, dans Ghost Cat Project, Fish Grikowsky (Alberta, 9) présente l'un des projets les plus interactifs. Après avoir « sculpté » des chats-fantômes en bois, il les donne aux visiteurs de l'expo ou à des amis en leur demandant de se faire photographier avec «leur» chat-fantôme et ensuite d'afficher leur photo sur un site internet précis, qui devient ainsi un musée virtuel. Le projet initial visait à rassembler au moins cent images pour ainsi créer une « histoire » faite d'images de chats-fantômes dans des contextes divers « lisibles/visibles » en la déroulant sur son appareil électronique (téléphone, ordinateur, etc.), un peu comme une BD digitale.

D’une manière plus traditionnelle, mais tout aussi interactive, l'œuvre de Charity Slobod (Alberta, 10), intitulée « Many Hands, Light work : The Forerun of Neil Gaiman », propose au lecteur-spectateur de créer sa propre case de BD à partir d'un extrait du script écrit par l'un des scénaristes de BD les plus célèbres, Gaiman (auteur entre autres de Sandman dans les années 1990 ; traduction 2000). Elle place des crayons et divers ustensiles de dessins (stylos, marqueurs, etc.) et une page A4 sur laquelle elle a dessiné un gaufrier sur une planche de $30 \mathrm{~cm}$ de haut et $20 \mathrm{~cm}$ de large qu'elle pose sur un trépied-chevalet, proposant ainsi au spectateur de devenir un « spectaCteur».

Voici les textes écrits par les artistes avec les mots clés pour accompagner leur œuvre (chacun suivi d'une photo de l'œuvre).

\section{1) « No Title » [Sans titre] des commissaires Julio Ludemir \& Victor Marcello}

Cinq auteurs de BD ont été invités à rencontrer cinq résidents des favelas [bidonvilles] de Babilonia et Chapeu Mangueira à Rio de Janeiro (Brésil). Chaque habitant a raconté sa propre histoire et sa relation avec son quartier, car ils viennent tous de familles qui ont aidé à fonder la communauté. D'une dame âgée qui dirige un groupe d'artisanes, à une jeune fille qui fut la première de sa famille à obtenir un diplôme universitaire, au fils d'un chef de favela et à un propriétaire de bar où beaucoup de célébrités sont passées, à travers cette conversation, ces gens ont montré avec fierté leurs origines par le biais de souvenirs intéressants et de dates importantes pour la communauté. Inspiré par ces histoires, chaque artiste a fait une BD de cinq pages. Cellesci ont été imprimées sur des posters qui à leur tour ont été affichés sur des murs des deux favelas et sur les panneaux de Copacabana Beach. La réaction des résidents pendant l'installation de ces posters fut la meilleure que l'on puisse espérer : ils ont reconnu les personnages réels qui ont pris 
part aux fondements de leurs communautés et ils se sont aussi montrés reconnaissants d'avoir été entendus et montrés sous leur meilleure apparence.

Artistes: Bruno Drummond, Daniel Og, Elodie Lacaze, Marilia Bruno, Tiago Elcerdo

Familles: David - famille Bispo, Alexandre « Gorila », Angolano et Zé das Flores - famille Alves, Edmilson et Jorge, Ana Gonçalves «Tina », Mme Augusta

Mots clés : Brésil - favela - intervention urbaine - collaborative - poster

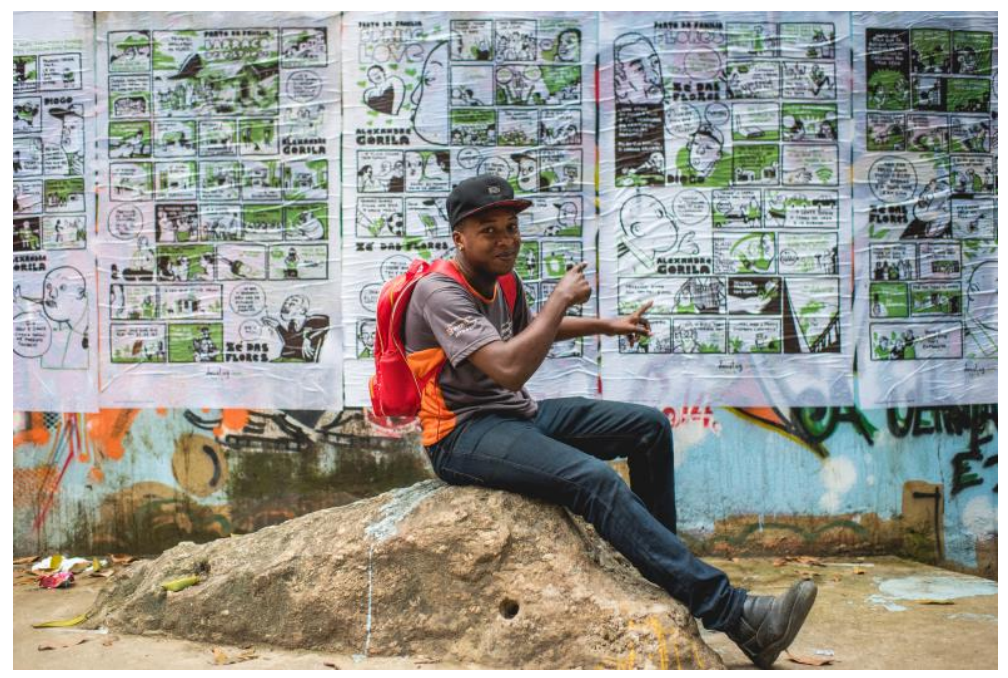

Cinq des six posters de l'histoire de Daniel Angolano avec Daniel lui-même sur la photo

(Permission des auteurs de l'exposition murale, Victor Marcello)

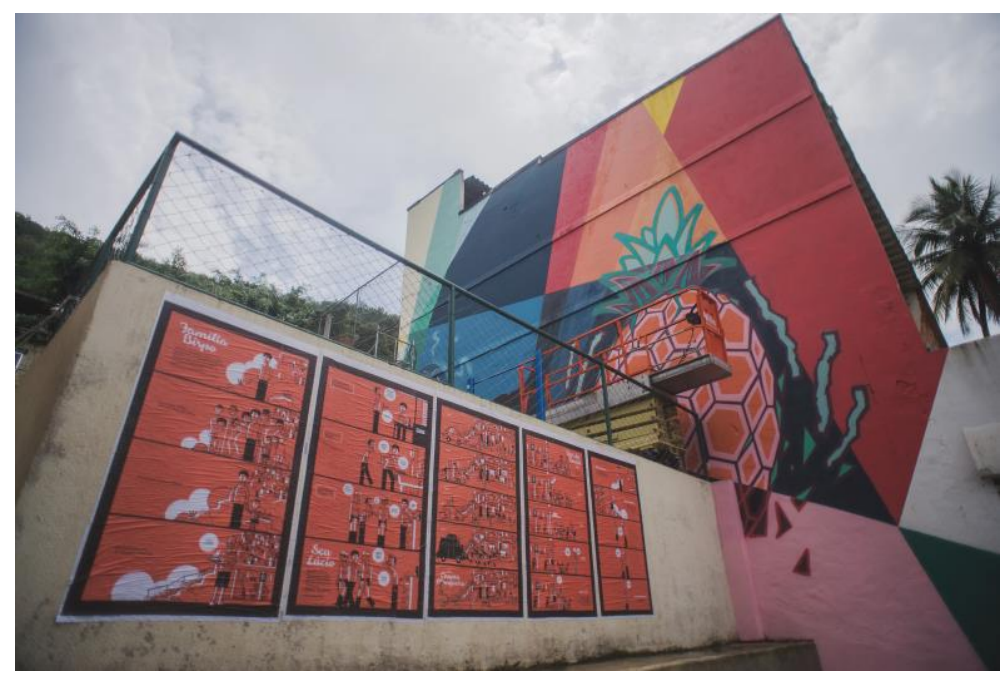

Poster mural géant réduit en poster A4 pour l'expo, voir image suivante. 


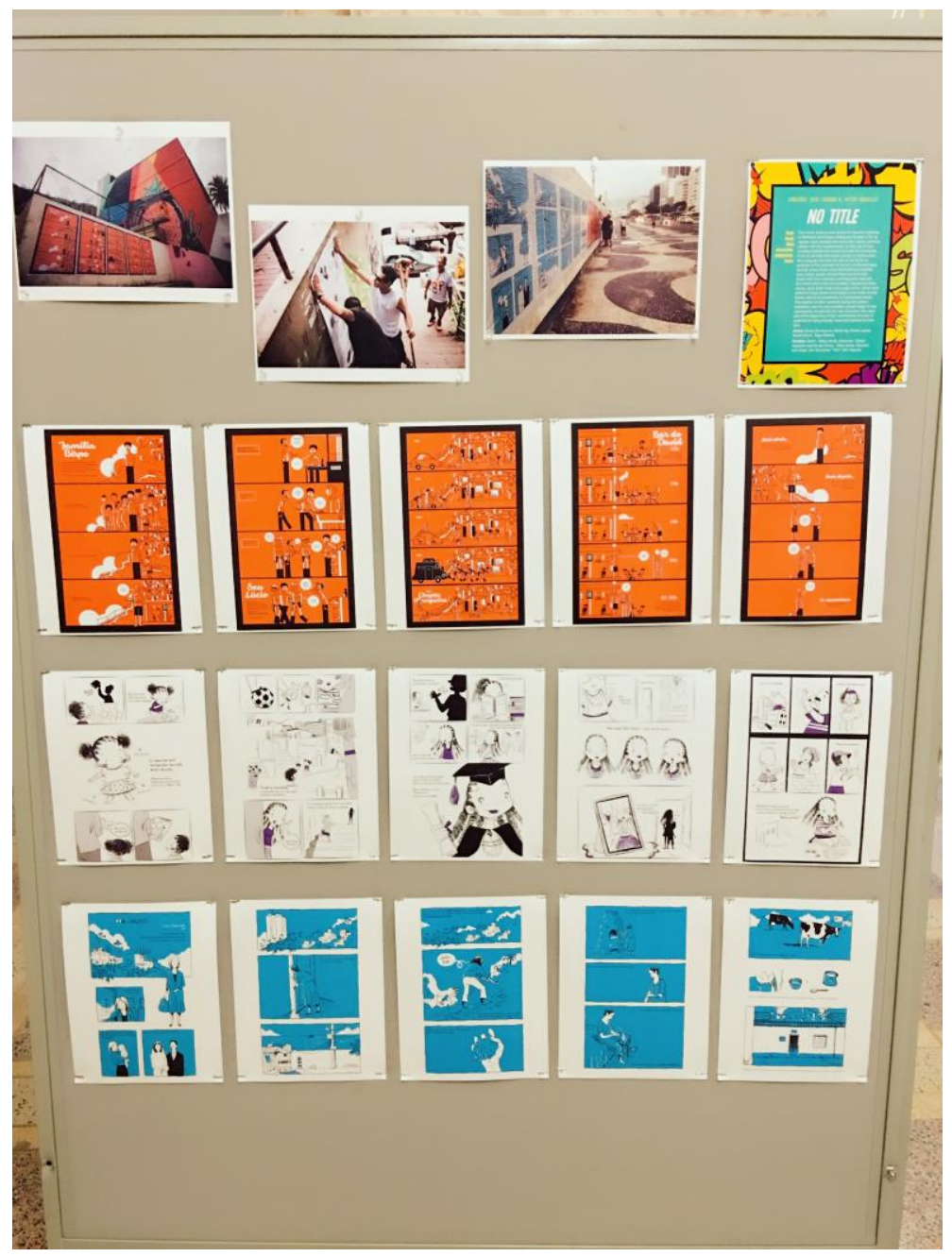

Deux des six séries de cinq posters ici réduits en posters A4 pour l'exposition intérieure (Permission des auteurs. VICTOR MARCELLO)

\section{2) « Mort par Pixel » de Galamot Shaku \& NAVA @LATINOTOONS}

«Mort par PiXEL » est une BD muette en trois couleurs ayant mûri avec le temps et se développant en un Gif-Codex animé sur les «morts-vivants ». Inspiré par la conception traditionnelle indigène sur le monde souterrain Mexica, le Mictlán, il se base sur une esthétique du jeu-vidéo en 8-bit, visuellement mêlé avec le tissage mésoaméricain et d'autres arts natifs américains. La projection de ce « comic » sur trois surfaces simultanées dans une forme narrative triptyque a pour but de dépasser les limitations d'un média mobile traditionnel, avec le public et leurs propres observations fermant le cercle comme le quatrième mur. Les artistes ont cherché à défier la manière dont l'utilisateur moderne peut interagir avec sa propre conception de la mortalité alors qu'il suit les aventures de leur héros en 8-bit “*” qui traverse neuf niveaux vers le jugement final aux mains du 
Señor de los Muertos [seigneurs des morts].

Conçu initialement pour TACO DE OJO, une anthologie de BD RISOgraph latino-américaine, il fut créé sur plusieurs mois avec le collectif Latino Toons, à travers une collaboration continue entre Galamot Shaku \& NAVA; son exécution s'inspire du comix underground, du GIF-Art, du design du jeu-vidéo, et de l'expérience VJing.

Mots-clés : Pixel, Codex, Comic, Animation

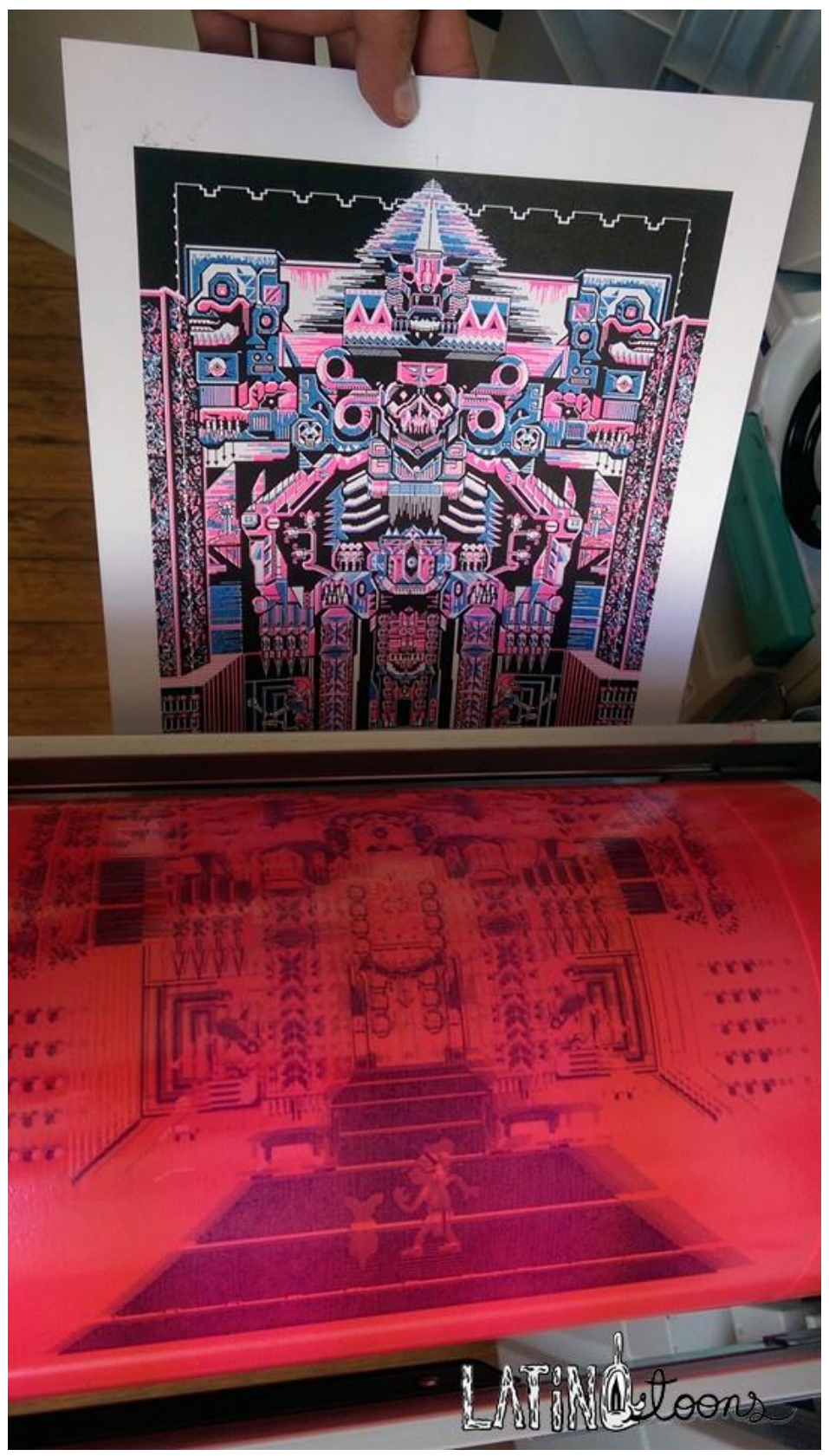

Image venant de la vidéo animée de « Death by Pixel » du collectif Latinotoons (Permission des auteurs Nava) 


\section{3) «Onomatopoeia Anthropomorphica » de Manuel Gómez Burns}

L'idée derrière cette série (que j'ai plus tard appelée «Onomatopoeia Anthropomorphica ») a commencé comme un simple jeu sur mon cahier d'esquisses en 2009. Elle est devenue un défi quand j'ai décidé de créer quelques séquences à partir de ces esquisses une année plus tard. J'ai créé un groupe de strips de quatre ou six panneaux/cadres, mais ce comic de six pages est la plus longue histoire de cette série jusqu'à présent. Dans ce cas, deux personnages de pupilles [« eyeball »] interagissent avec un personnage-onomatopée qui est le vilain de cette histoire. Après avoir terminé quatre pages, cela m'a encore pris plusieurs mois pour finir ce comic.

Une partie du travail que je fais comme cartooniste est d'essayer de jouer avec quelques éléments communément trouvés dans les comics (dans l'animation aussi) pour créer une séquence amusante et absurde. Je suppose que la manière dont je présente mon travail pour cette exhibition, est quelque peu traditionnelle (encre et brosse sur papier), mais le personnage n'est pas habituel. Maintenant, il y a aussi un autre élément qui est lié avec l'exposition : ce comic n'a pas de dialogue ou de texte sauf pour le titre, et même si le personnage-onomatopée est en anglais, les gens qui visitent l'exposition et qui ne parlent pas anglais, peuvent comprendre l'histoire.

Mots-clés : Expérimental, cartoon, absurde

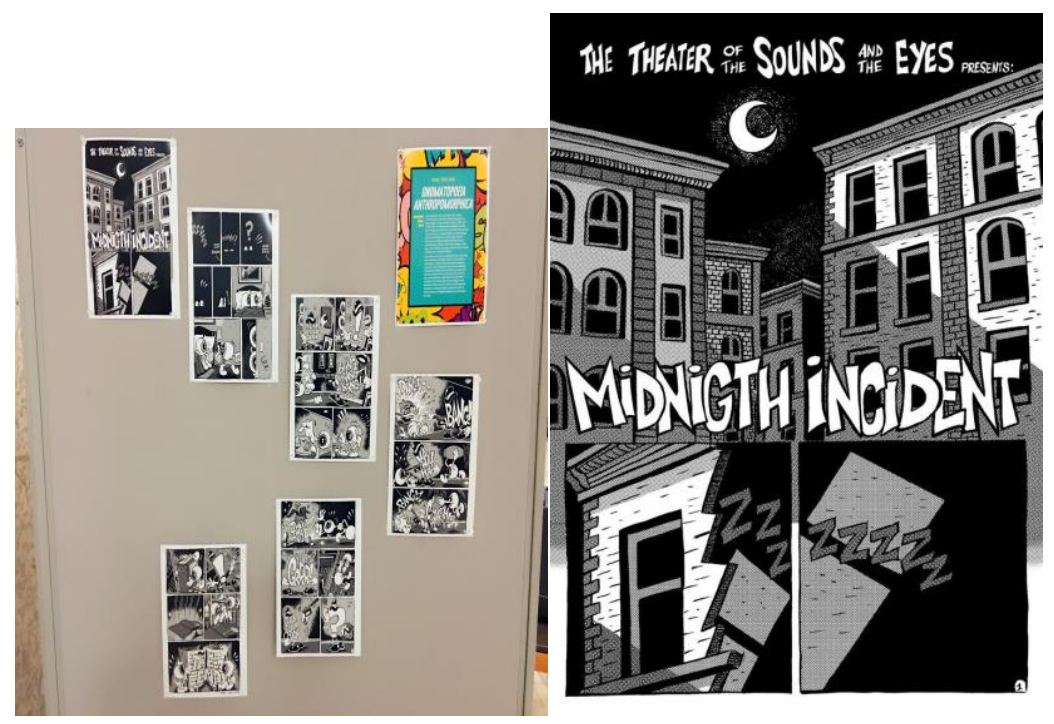

Vue des six pages en A4 posées sur le mur pour l'exposition avec le texte explicatif à côté et $1^{\mathrm{e}}$ page des six pages de l'histoire sur poster A4 (Permission de l'auteur Manuel Gómez Burns) 


\section{4) « Comic Splits » du collectif In Wonder}

Récit animé à projeter au mur, Comic Splits propose une relecture de l'esthétique du split screen comme relevant de la bande dessinée. L'œuvre a été conçue par le collectif In Wonder, composé d'Emmanuel Espinasse, Henri Lemahieu, Régis Pinault et Johanna Schipper, et s'inscrit dans une série de recherches menées par le collectif sur la bande dessinée numérique et l'art digital. L'écran, comme nouvel espace de lecture et de visionnage, est devenu depuis une quinzaine d'années un moyen de publication, de diffusion, et de création pour la bande dessinée. Pour In Wonder, ce nouvel espace oblige à repenser la bande dessinée à partir des possibilités offertes par l'univers numérique (animations, interactions et sonorisations) sans rien perdre de sa cohérence. La mutation qui s'opère en passant du papier à l'écran, peut englober la question du déploiement de la bande dessinée dans des lieux physiques, notamment le musée ou la salle d'exposition. Dans Comic Splits, comme dans La table des opérations, In Wonder développe un récit à partir d'un ensemble d'icônes qu'elle a conçu. La permutation spatiale des cases complexifie encore le dispositif, instituant l'écran comme terrain de jeu occupé par des forces en déplacement. En alternant linéarité et libre association, la narration de Comic Splits se veut expérimentale et tend à introduire la dimension du mouvement dans le processus de lecture. www.in-wonder.com

Mots-clés : Animation, projection, bande dessinée numérique

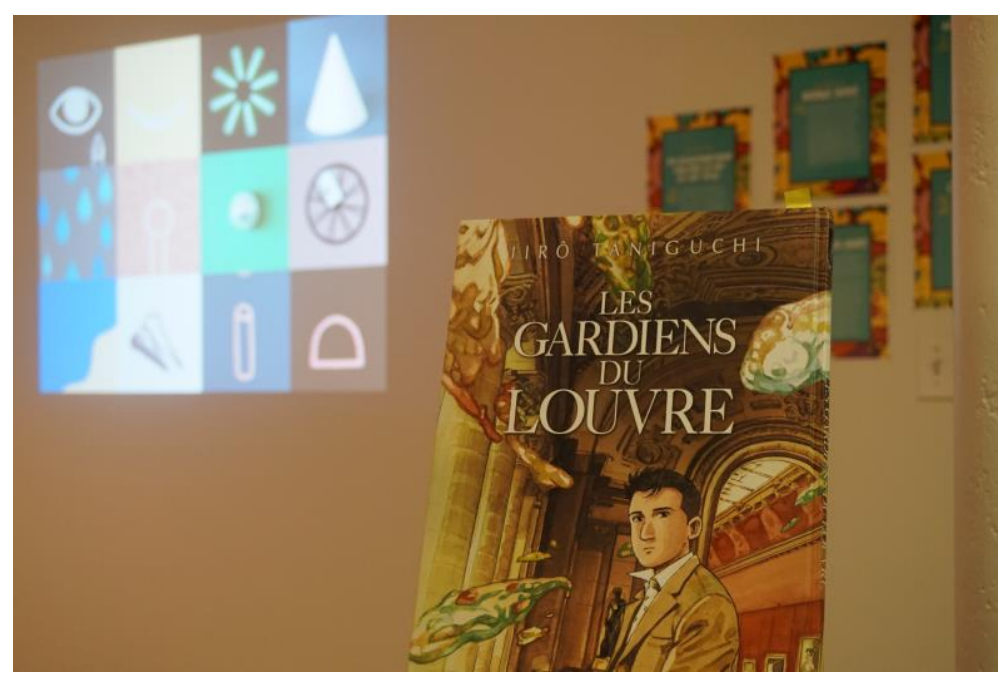

A gauche, projection sur le mur de la vidéo « Comic Splits » du collectif In Wonder. (Permission des auteurs du Collectif) 


\section{5) La table des opérations}

Livre pop-up, jeu d'échec pour un seul joueur, planche de bande dessinée à lire à plat et en volume : La table des opérations est de nature hybride. Elle a été conçue par le collectif In Wonder, composé d'Emmanuel Espinasse, Henri Lemahieu, Régis Pinault et Johanna Schipper. Les travaux d'In Wonder explorent la zone frontière où la bande dessinée (comme système graphique et narratif) vient rencontrer l'art contemporain (comme corpus conceptuel et formel). Les travaux d'In Wonder n'ont pas pour but de faire sortir la bande dessinée du livre, mais partent du principe que le livre n'est pas l'unique espace dédié à la bande dessinée et que son langage nourrit nombre de formes actuelles dans les arts visuels. Les œuvres d'artistes ou designers, comme Matt Mullican, Robert Morris ou Charles \& Ray Eames, pourraient, selon In Wonder, relever d'une bande dessinée non linéaire conçue pour et dans l'espace. À partir de ce postulat, In Wonder a développé un vocabulaire polysémique, comportant un certain nombre d'icônes pouvant devenir tantôt personnages, tantôt signes, afin de déployer des récits sur différents supports. Le plateau du jeu d'échecs est l'un des supports choisis par In Wonder pour la richesse de sa connotation. L'œuvre Comic Splits, jouant sur l'hybridation entre bande dessinée et cinéma d'animation, lui vient en écho.

\section{Mots-clés : Bande dessinée, pop-up, jeu}

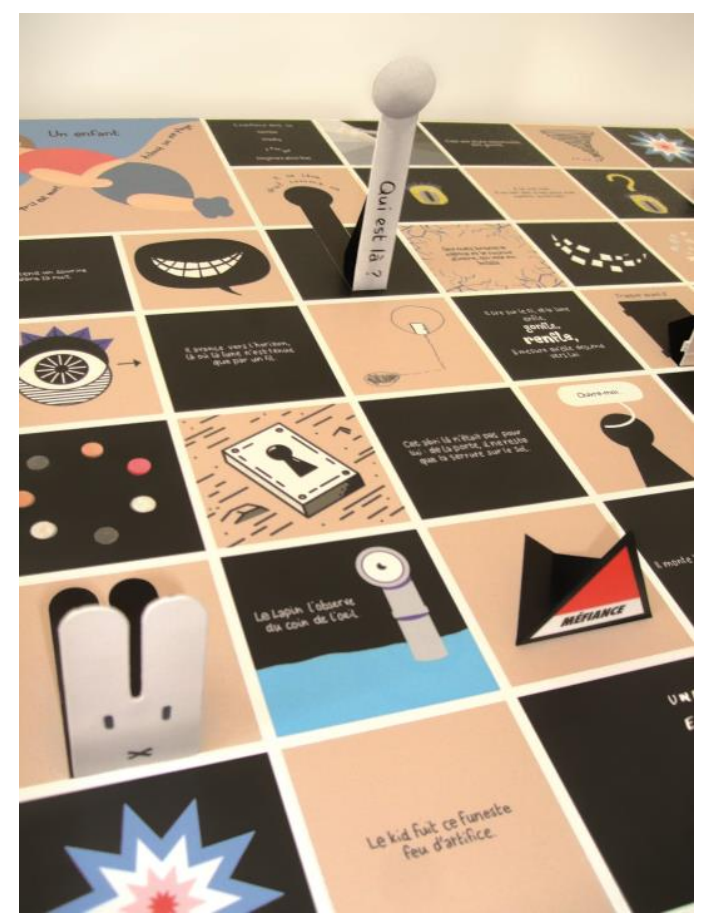

Photo de la Table d'opérations du Collectif In Wonder (Permission des auteurs du Collectif) 


\section{6) «Le projet Chats-fantômes » [« Ghost Cat Project »] de Fish Griwkowsky}

En utilisant des chats sculptés et peints sur du contreplaqué, le projet « chats-fantômes » s'amuse avec les idées de la $\mathrm{BD}$ et de l'espace de la galerie tout en voguant digitalement grâce à hashtag de Instagram. Chaque « chat » est donné à une personne différente qui, en échange, le photographie, le hashtague \#99ghostscats et le partage sur les médias sociaux. Deux cadres - le premier et le dernier - sont pendus dans la galerie, tandis qu'un écran montre les œuvres d'art hashtaguées par les individus qui ont pris les chats-fantômes en photo. Le projet a donc techniquement cent artistes. Ghostcats est apparu sur En masse Underpass et dans le livre SNAP. De nombreux chats-fantômes ont déjà été faits et distribués, y compris à Paris, Bruxelles et Toronto.

\section{Mots-clés : sculpture, peinture, acrylique sur contre-plaqué, internet, hashtag}

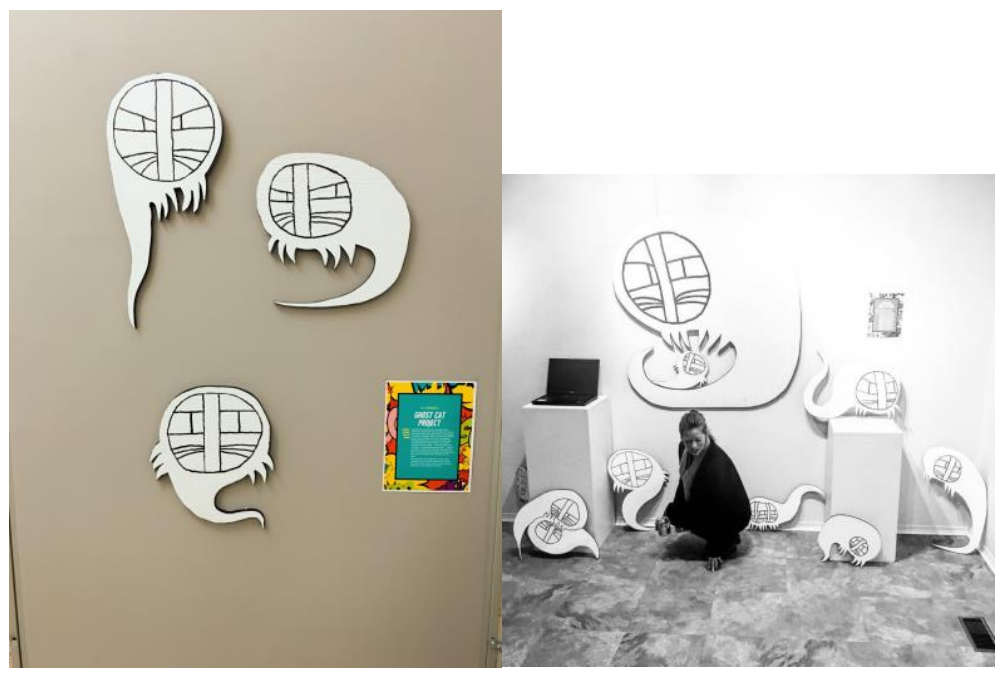

Trois « chats » accrochés au mur venant du « Projet Chats-Fantômes » pour l'exposition dans Rutherford à l'Université de l'Alberta

Les chats-fantômes exhibés dans la galerie CAVA (Permission de l'auteur Fish Griwkowsky)

\section{7) «'Pop-up' Culture » de Larisa Sembaliuk Cheladyn}

Dans un âge où les médias sociaux se multiplient et le degré d'attention décroit, auteur et illustrateur sont constamment défiés par les moyens disponibles pour se connecter avec leur public de lecteurs. Utilisant un devoir donné dans mon cours MLCS 399 avec le professeur C. ReynsChikuma, j'ai adapté et illustré la nouvelle d'Alice Monroe intitulée « Boys \& Girls » [Garçons et filles]. Le critère que je me suis imposé était de modifier l'histoire pour des lecteurs d'école élémentaire et ensuite de la formater dans un écran pop-up d'un seul cadre pour une exposition publique dans un endroit à haute circulation ${ }^{2}$. 
Mots-clés : Imprimé sur pop-up rétractable, crayon, encre et craie colorée sur papier-ponce

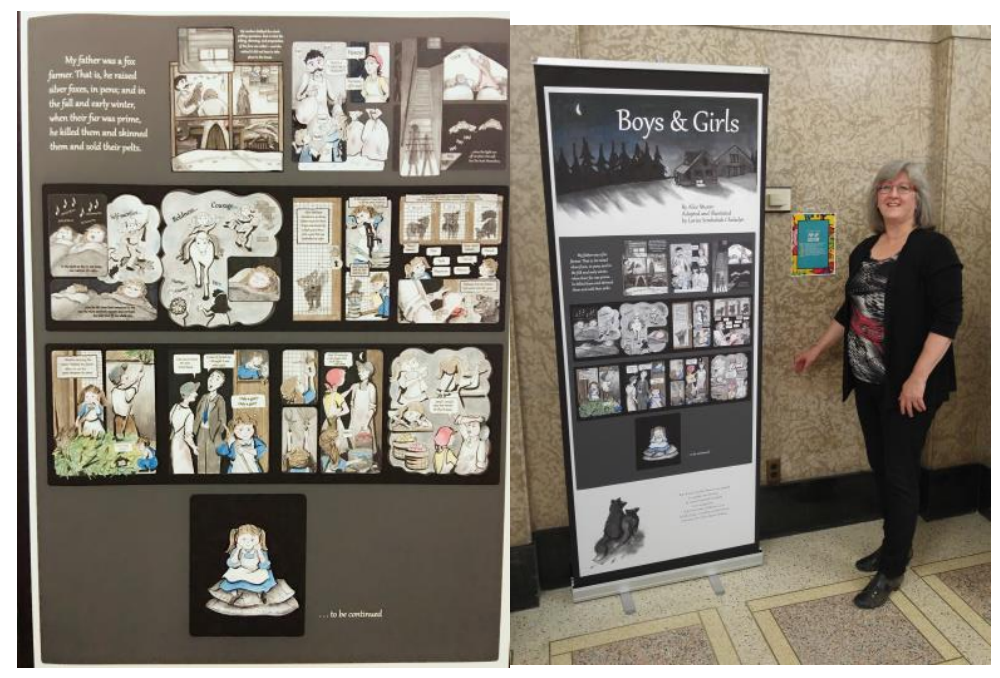

Deux photos du pop-up de Larisa Sembaliuk-Cheladyn (Permission de l'auteure)

\section{8) Auto-portrait d'un/e Albertain/e (Self Portrait of an Albertan) de Chelsey Campbell}

Self Portrait of an Albertan utilise des éléments comiques pour illustrer la complexité et l'ambivalence de la vie contemporaine en Alberta. M'inspirant de dessins éditoriaux, ce travail utilise un format de cadre unique et l'hyperbole de la satire politique pour créer un monde grossier et exagéré de la consommation. Dans l'œuvre, l'individu apparait comme un marginal à la fois critique et complaisant envers le système industriel, reflétant le propre combat de l'artiste avec cette dualité. Rompant avec la tradition de l'outil textuel dans la BD comme la bulle, le récitatif ou l'onomatopée, Self Portrait of an Albertan repose sur le titre pour apporter le message politique. La séparation de l'illustration et du commentaire politique enveloppe le public dans le monde ; on est à la fois outsider quand on est séparé du message politique évident et complice fidèle. Self Portrait of an Albertan repose sur la capacité du cartoon à explorer des sujets politiques souvent grotesques et inconfortables, défiant le public pour explorer l'absurdité de sa propre relation individuelle avec l'industrie et la consommation.

Mots-clés : Illustration, dessin, encre 


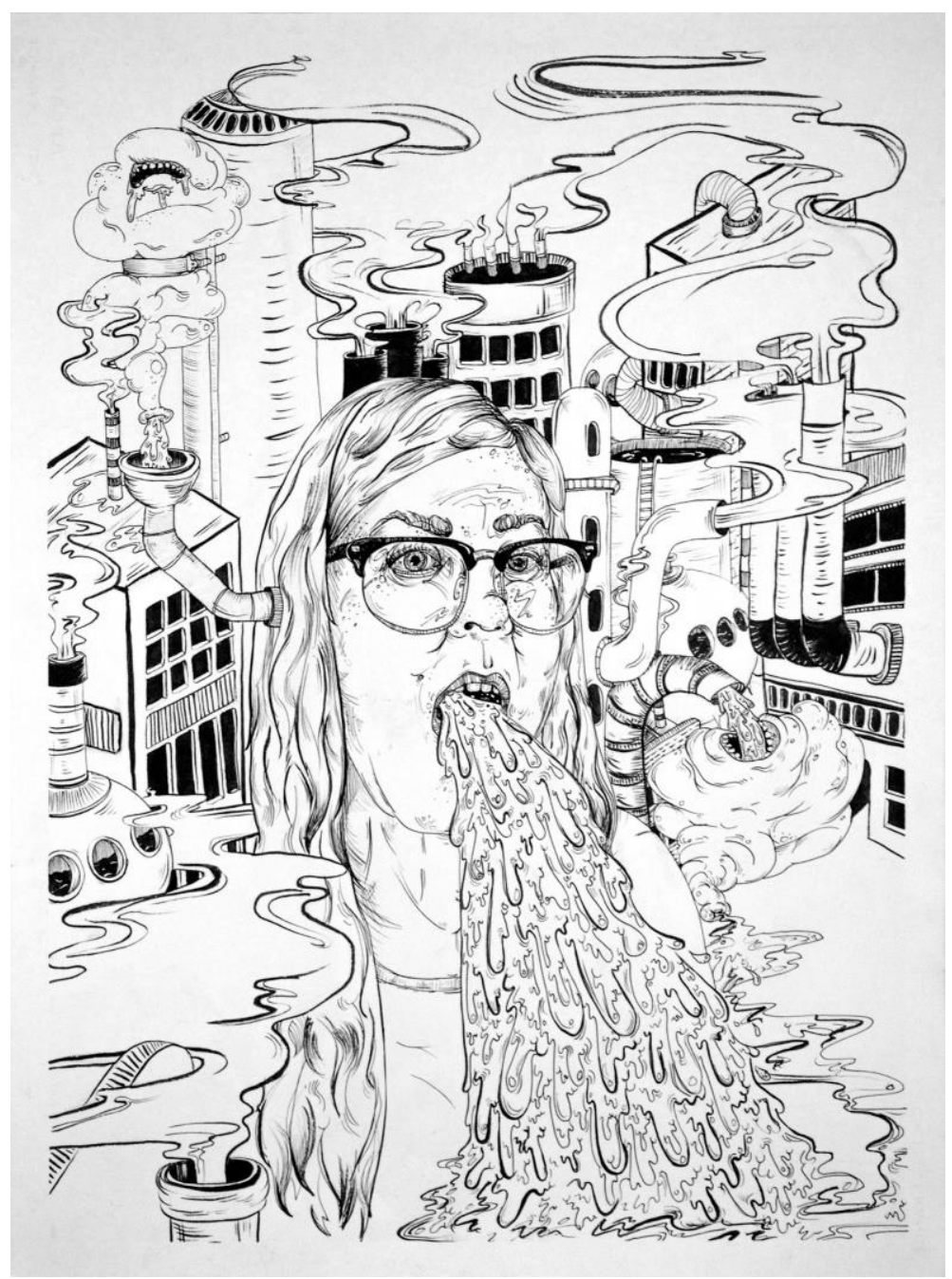

Photo du dessin de Chelsea Campbell (Permission de l'auteure)

\section{9) «WORMS » [Vers] de Simone Kousol-Graham}

Dans une société globalisée dominée par le storytelling [récit] imagé, le visuel est la manière la plus avancée pour diffuser l'information à de larges segments de la population. A travers la BD, l'imagerie séquentielle connotant le temps et l'espace permet de raconter une histoire de l'auteur d'une manière qui fait l'usage de visuels en combinaison avec le texte. A travers ce mode de storytelling, les lecteurs peuvent aisément comprendre une séquence d'événements, similaires aux films ou animations. Ceci peut aussi être dit d'un musée, puisqu'il y a un arrangement d'œuvres et d'artefacts organisés qui fait la chronique de certains points dans le temps. Ce lien narratif entre comics et musée aide les lecteurs à mieux comprendre la dissémination de l'information et de la compréhension cognitive. Avec l'animation «WORMS », imagerie, vidéo et son s'unifient pour 
personnifier notre bon ami le ver. A travers une histoire séquentielle et visuelle, les spectateurs deviennent conscients de la relation particulière qui existe entre l'humain et les vers.

Mots-clés : Animation, illustration, vidéo

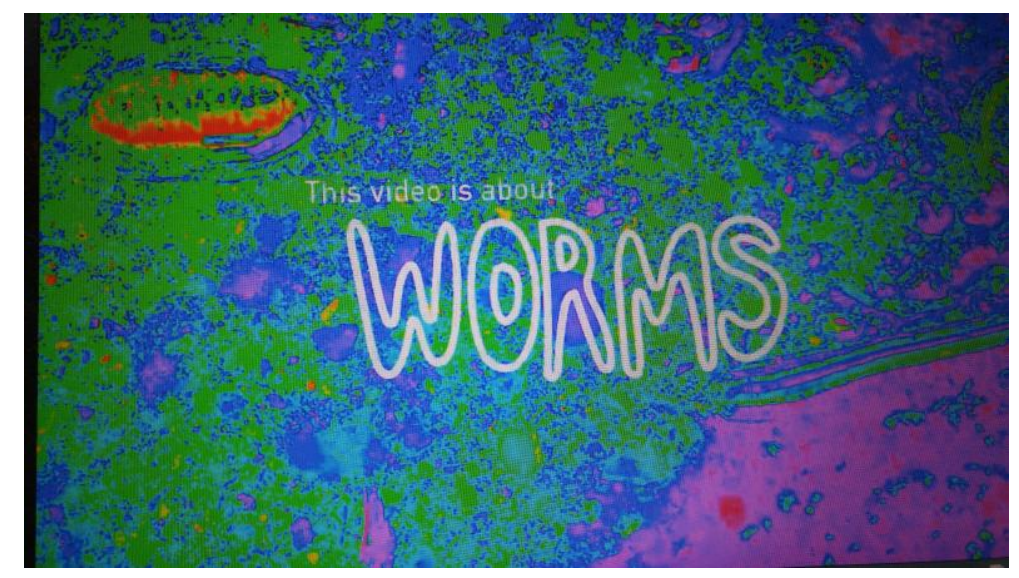

Photo extraite de la vidéo «Worms » [vers] de Simone Kousol-Graham (Permission de l'auteure)

\section{0) «Oh les beaux jours ! » de Christian Quesnel, Mélanie River et Evelyn Accad}

«Oh les beaux jours ! » est une bande dessinée multimédia, une première expérience pour les artistes Mélanie Rivet et Christian Quesnel. Originalement publiée dans la collection «Un trait d'humanisme » (éditions Neige-galerie, 2015) en réaction aux attentats à Charlie Hebdo, cette vidéo BD est un pastiche du style théâtral de Beckett et dénonce le climat de terreur instauré par l'État Islamique sur les territoires qu'il occupe en Irak et en Syrie.

Christian Quesnel, qui est artiste en bande dessinée, assume ici l'univers sonore de ce court récit, ajoutant à sa pratique un nouveau moyen d'expression par le son et quelques mouvements de caméra, venant amplifier le réalisme du personnage de la femme (la voix en arabe, d'Evelyne Accad, écrivaine libanaise) et de son environnement (le vent). Quesnel questionne depuis quelques années les différentes formes que peut prendre la bande dessinée à l'extérieur du livre standard imprimé et notamment dans la mise en exposition de celle-ci. Outre le son, l'espace inter iconique est ici transposé en coupes progressives d'une image à l'autre, ajoutant volontairement un aspect vaporeux au récit, tentant de préserver cet espace où le lecteur effectue le lien mental entre les images. Cette démarche est aussi l'occasion pour Quesnel d'apprivoiser un récit dont la case est uniforme, d'y mesurer les possibilités et les limites.

Mots-clés : Vidéo, BD narrative 


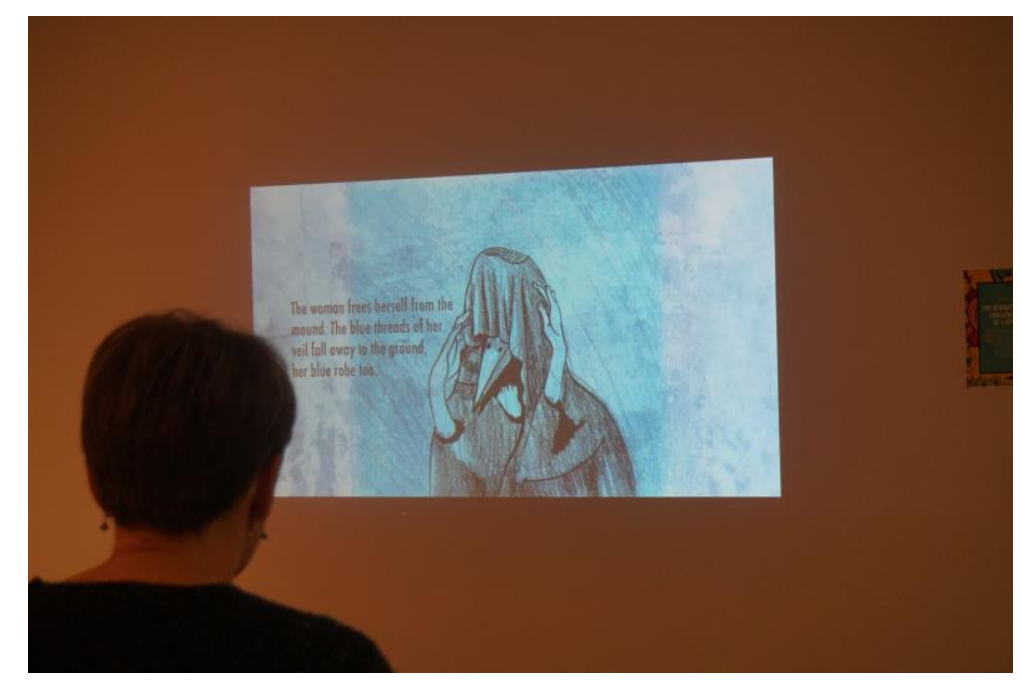

Vidéo de Christian Quesnel projetée sur le mur (Permission de l'auteur)

\section{1) «Mains nombreuses, tâche aisée : préparer le travail de Neil Gaiman » de Charity}

\section{Slobod}

Des mots aux images, puis aux cadres, ou autrement dit «texte, image et narration », ces trois étapes marquent les différents modes de la BD. Que diriez-vous si ces formes distinctes en BD étaient parfois (souvent ?) créées par plusieurs artistes, par plus de deux mains ? Cela commencerait avec une histoire venant de l'auteur, écrivant des mots pour décrire des représentations vues seulement par son esprit. Ce scénario serait ensuite passé à un artiste pour le rendre visuel. Le média de cet artiste pourrait n'utiliser que le crayon ; et donc un encreur serait nécessaire pour solidifier le dessin esquissé. Si la couleur est requise, un coloriste serait aussi nécessaire ! Et si ces personnages étaient amenés à parler ? On aurait alors besoin d'un lettreur pour les faire dialoguer. Juste pour un seul cadre, cela peut prendre plusieurs heures de plusieurs interprètes artistiques. La question serait alors : 1'intention originale de l'auteur se serait-elle modifiée en passant à travers tant de mains ?

Ici, spectateurs, nous te mettons au défi en te proposant de prendre part à la création d'un cadre de BD. Dans cette partie de cette exposition, nous te proposons de lire le scénario original de la BD de Neil Gaiman, Sandman : Season of Mist v.4 (Sandman [le marchand de sable] : saison de brume v.4). Essaie-toi à esquisser, encrer, colorer et lettrer dans un cadre ci-dessous (tu peux déplacer le carton-support et t'asseoir). Une fois ton interprétation visuelle finie, retourne le support et regarde 
le dessin original pour voir si tu es près de la vision originale de l'auteur. Les grands esprits visualisent-ils de la même manière ? ${ }^{3}$

Mots-clés : Interactivité, cadre sur poster, effaçable, chevalet

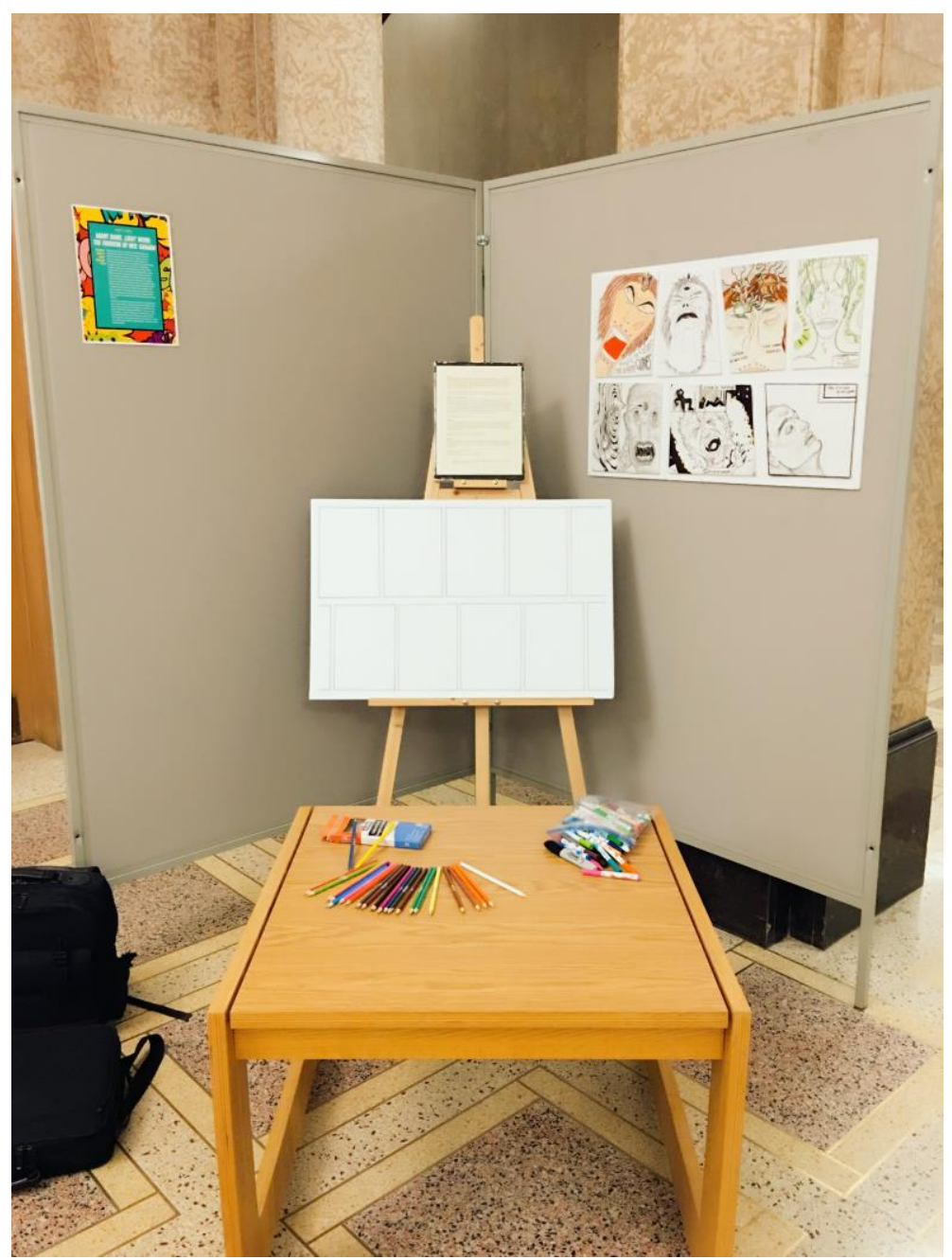

Photo de l'espace créé par Charity Slobod pour permettre aux spectaCteurs et spectaCtrices de créer leur propre BD à partir de l'extrait du script de Gaiman (Photo de l'auteure ; permission de l'auteure)

\footnotetext{
NOTES

${ }^{1}$ Ce postulat du sens unique de la BD est remise en question de manière intéressante par Ian Hague dans Comics and the Senses : A Multisensory Approach to Comics and Graphic Novels, Routledge, 2014.

${ }^{2}$ Larisa Sembaliuk Cheladyn a en fait présenté un second projet intitulé « The Gesamtkunstwerk Challenge » [le défi de l'art total]. Basé sur les défis qui se présentent lorsque l'on enseigne l'illustration de roman graphique, pour ce projet j'ai choisi de soumettre deux histoires illustrées faites par des étudiants inscrits en Art 211-Illustration à Kings University à Edmonton en 2012-13. Les objectifs de cette classe étaient d'explorer l'illustration du roman graphique en équipe en incluant le développement de personnages, une minimisation du texte et la construction du décor. Chaque histoire a été téléchargée sur un format «power point » pour faciliter le partage digital en ligne sur un écran d'ordinateur. Mots-clés : Médias mixtes, collaboration de multiples artistes.

${ }^{3}$ De gauche au-dessus à droite en-dessous :

Cadre 1 : Dessinateur : Vonn Gondziola ; Encreur/coloriste : Charity Slobod ; Lettreur : Rylan Kafara
} 
Cadre 2 : Dessinateur/encreur : Madeleine Mackay ; Lettreur : Rylan Kafara

Cadre 3 : Dessinateur : Marynek Howe ; Encreur/coloriste/lettreur : Rachael Roberts

Cadre 4 : Dessinateur : Rachael Roberts ; Encreur/coloriste : Charity Slobod ; Lettreur : Rylan Kafara

Cadre 5 : CEuvre complète par Marynek Howe

Cadre 6 : Euvre complète par Ian Cool

Cadre 7 : Dessinateur : Lauren Bell ; Encreur/lettreur : Charity Slobod 